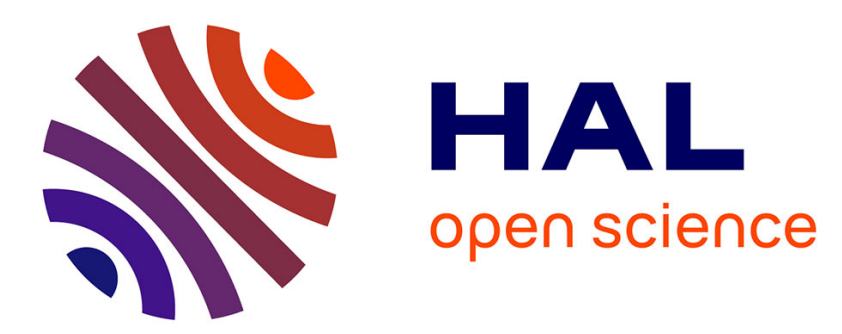

\title{
Adaptive genetic algorithm-based approach to improve the synthesis of two-dimensional finite impulse response filters
}

Kamal Boudjelaba, Frédéric Ros, Djamel Chikouche

\section{- To cite this version:}

Kamal Boudjelaba, Frédéric Ros, Djamel Chikouche. Adaptive genetic algorithm-based approach to improve the synthesis of two-dimensional finite impulse response filters. IET Signal Processing, 2014, 8 (5), pp.429 - 446. 10.1049/iet-spr.2013.0005 . hal-01076967

\section{HAL Id: hal-01076967 https://hal.science/hal-01076967}

Submitted on 23 Oct 2014

HAL is a multi-disciplinary open access archive for the deposit and dissemination of scientific research documents, whether they are published or not. The documents may come from teaching and research institutions in France or abroad, or from public or private research centers.
L'archive ouverte pluridisciplinaire $\mathbf{H A L}$, est destinée au dépôt et à la diffusion de documents scientifiques de niveau recherche, publiés ou non, émanant des établissements d'enseignement et de recherche français ou étrangers, des laboratoires publics ou privés.

$$
\text { Copyright }
$$




\title{
Adaptive genetic algorithm-based approach to improve the synthesis of two-dimensional finite impulse response filters
}

\author{
Kamal Boudjelaba ${ }^{1}$, Frédéric Ros ${ }^{1}$, Djamel Chikouche ${ }^{2}$ \\ Email: kamal.boudjelaba@univ-orleans.fr, frederic.ros@univ-orleans.fr,dj_chikou@yahoo.fr \\ 1. Prisme Laboratory, IRAuS Pole, Axis Image and vision, Polytech'Orléans, 12 rue de Blois, Orléans, France \\ 2. Electronics Department, University of M'sila, Rue Ichebilia, M'sila, Algeria
}

\begin{abstract}
The design of finite impulse response (FIR) filters can be formulated as a non-linear optimization problem reputed to be difficult for conventional approaches. The constraints are high and a large number of parameters have to be estimated, especially when dealing with 2-D FIR filters. In order to improve the performance of conventional approaches, we explore several stochastic methodologies capable of handling large spaces. We specifically propose a new genetic algorithm in which some innovative concepts are introduced to improve the convergence and make its use easier for practitioners. The algorithm is globally improved by adapting the mutation and crossover and selection operators with the genetic advances. A dynamic ranking selection scheme is introduced to limit the promotion of extraordinary chromosomes. A refreshing mechanism is investigated to manage the tradeoff between diversity and elitism. The key point of our approach stems from the capacity of the genetic algorithm (GA) to adapt the genetic operators during the genetic life while remaining simple and easy to implement. Most of parameters and operators are changed by the GA itself. From an initial calibration, the GA performs the design problem while calibrating and repeatedly re-calibrating itself for solving it. We demonstrate on various cases of filter design a significant improvement in performance.
\end{abstract}

Keywords: Adaptive GA approach, Real-valued chromosomes, 2-D FIR digital filter, Tabu search, Simulated annealing, Hill-climbing.

\section{Introduction}

In recent years, with the rapid improvement in computer technology, two dimensional (2-D) digital signal processing has become more important. In particular, the design of 2-D digital filters has received a great deal of attention in various domains. They have been widely used for processing aerial and satellite photos, geophysical and seismological data, or geological and medical images. Digital filters can be classified into two groups: finite impulse response (FIR) filters [1] and infinite impulse response (IIR) filters. Since FIR digital filters are inherently stable and can have a linear phase, they are often preferred over IIR filters as they possess several desirable characteristics [2], e.g., guaranteed stability and absence of phase distortion. However, the number of independent filter coefficients required for optimally designing a 2-D FIR filter is generally large for practical applications. This makes efficient designs complex and highlights the need for more efficient techniques for designing 2-D FIR linear-phase filters [3].

The problem of filter design is to find a realization of the filter which meets each of the requirements to a sufficient degree to make it useful. The techniques for designing 2-D FIR digital filters have been developed extensively for several years [2,4-6]. The results of most of these techniques are given in the form of the impulse response of a 2D filter, so the designed filter is suitable for a direct convolution realization. Different methods can be used to find the coefficients from frequency specifications: windowing design, frequency sampling, weighted least squares design, or equiripple design [5,7]. The possible drawback to filters designed this way is that they contain many small ripples in the pass band, since such a filter minimizes the peak error. Most methods do not allow independent control of band specifications, in particular the transition bandwidth. Conventional methods for designing 2-D FIR filters using window functions and frequency sampling require a high filter order, and accurate control of the cutoff frequencies of the pass-band and stop-band remains difficult. In practice, these methods are too limited [8,9].

New design approaches have been investigated and have obtained interesting results compared to the abovementioned approaches. For example, the Remez method is based on a linear programming algorithm $[3,10,11]$. 
While interesting, the results are limited, since the difficulty then becomes one of converging the minimax solution. Unfortunately, it is known that for some functions the Remez method can lead to divergent behavior, even when the initial starting approximation is quite good. The weight least square (WLS) algorithm also provides some improvements, and can acquire analytical solutions. It suffers, however, from the drawback that the approximation accuracy deteriorates considerably near the band edges. Conversely, it must calculate an inverse matrix which can be computationally problematic when the filter degree is very high.

More recently, several joint optimizations [3] have been developed. The study in [3] deals with the minimax design problem of two-dimensional (2-D) linear-phase FIR digital filters with continuous and powers-of-two (POT) coefficients. In this study, the minimax continuous-coefficient design problem is expressed as a linear programming problem with inequality constraints. A number of successful and firmly established methods of 2-D digital FIR filter design and its complex associations have been proposed (e.g., [3,12]).

The design of 2-D FIR filters is an intensively-researched area, aiming at obtaining more general and innovative techniques that are able to tackle new and complex engineering problems of great relevance today. Evolutionary and genetic algorithms (GA) can be an interesting alternative for filter design. A wide variety of fields have published tutorial articles and computational studies documenting successes by evolutionary algorithms in extending the frontier of problems that can be handled effectively. GAs have been proven to be more efficient and attractive in finding the optimal or near-optimal solutions than conventional algorithms.

More generally, alternative approaches, such as neural networks, genetic algorithms, and other tools related to computational intelligence [12-15], have been devoted to the synthesis of design methods capable of satisfying constraints which would be unattainable if treated with the aforementioned conventional techniques. In some cases, such initiatives were successful and showed better performance indices than the conventional approaches. Some evolutionary filter design methods have been implemented $[2,8,13,19,20]$ for about ten years with promising results. We have already investigated Genetic Algorithms for designing 1-D and 2-D FIR filters [16-18] by developing dedicated genetic algorithms for the synthesis of 1-D and 2-D FIR filters. We demonstrated that by adding some flexibility in classical GA versions, ripples can be significantly reduced compared to the traditional and genetic approaches [13].

However, there are a few weak points associated with evolutionary methods, such as increased computational cost and the nonexistence of theoretical proof of convergence to a global optimum in sufficiently general conditions [9]. While these drawbacks are not foregrounded in published work, applying GAs for a dedicated problem remains difficult for practitioners who are often skeptical concerning GAs. There is no systematic process to find an efficient balance between the exploration and exploitation abilities of the search during the run. Most importantly, the amount of simultaneous parameterization and the tuning of several parameters can often lead to extremely high computation costs without ensuring good results. For more than 10 years, many promising studies [21] coming from the pattern recognition community have focused on parameter adaptation (mutation, crossover rates and population sizes) and on the proposals of new variants (e.g. intelligent crossover, elitist recombination) to tackle these well-known general limitations. GAs applied to handle filter design problems could take more advantage of these advances.

More generally, algorithms that use artificial intelligence techniques, i.e. evolutionary algorithms (EA), tabu search (TS) [22-24], simulated annealing (SA) [25-27] and the hill-climbing method (HC) [28-30], have received increasing attention in the field of global numerical optimization. They have been successfully applied in a wide variety of areas. However, they cannot be effective for all types of optimization problems. To solve a complex optimization problem, the most effective way is to design a tailor-made algorithm that suits the specific need. Consequently, there is a need to search for more pervasive methods, capable of overcoming such weaknesses.

There are literally several hundreds of relevant papers representing a broad sampling of the usage of genetic algorithms in the literature over the past 20 years and cover a wide variety of topics. However, GAs differ greatly in the amount of tuning needed to reach a given performance, and this tuning cost depends on the overall setup of the GA that directly depends on the time and effort one can permit to tune the GAs. Throughout the relevant literature, one can see that the cost of tuning parameters is largely ignored. Adaption and flexibility in GAs is currently one of the most important and promising areas of research in evolutionary computation.

This paper is in line with the current challenge. It presents alternative approaches that take advantage of the power of the well-known stochastic global optimization technique called genetic algorithm and its application to the synthesis of 2-D FIR filters. The aim is to present a consistent engineering approach to the formal specificationdriven optimal design of 2-D FIR filters. The design task of 2-D non-recursive filters is formulated as a constrained 
optimization problem. Although the algorithm is suitable, with minor adaptations, for applications to any kind of 2-D FIR filter, we have chosen to focus on real-coefficient FIR filters, in view of their importance in engineering practice and their inherent properties (linear phase, stability, flexibility and easy implementation).

Using the genetic algorithm, promising results are shown here in designing 2-D FIR filters. The optimization process is mainly governed by genetic operators that perform crossover and adaptive mutation whose initial principles are based on the best recent advances coming from the pattern recognition community.

For a class of problems and the associated know-how, GAs can perform robustly under a range of parameters that are pre-calibrated as starting points to work from. The main novelty is the integration of several dedicated and complementary mechanisms to assist the GA convergence for a more efficient design and to enable practical use by non-experts with different configurations (low pass, high pass, band pass and band cut). These mechanisms afford real flexibility and simplicity for the user as they enable the GA to be calibrated and repeatedly re-calibrating itself. The GA relevance is achieved through the strategy and not through initial parameters that play a second role.

Furthermore, their computational costs are highly optimized. Then, the user only needs to enter the filter specifications follow a set of input parameters specify a few extra parameters, without being faced with all the evolutionary computation complexity. By combining the know-how of filter design and best advances in GA design, we have obtained a so-called "free algorithm" that optimizes the GA usability.

To the best of our knowledge, there to be no reported GA approaches which go so far in this direction to achieve the fast design of efficient 2D FIR filters.

The paper is set out as follows: Section 2 briefly describes the characteristics of digital filter design and its reformulation as a constrained minimization problem. In section 3 , we introduce the proposed adaptive genetic algorithm (AGA) and explain the different mechanisms implemented in order to improve the efficiency of native versions. To illustrate the effectiveness of the proposed GA and evolutionary approaches, design examples are included in Section 4. Our results are compared with those from established methods for FIR digital filter design and other classical algorithms such as the windowing method. They are also compared to the most popular GAs dedicated to filter design [13,31]. Finally, Section 5 reports some concluding remarks and presents directions for further research.

\section{Design procedure of 2-D FIR filters}

The design of digital filters, as with most engineering tasks, is a multistage iterative process. The key stages are filter specification, coefficient computation and structure realization. An attractive goal is to perform the optimization by several approaches in order to seek out the filter design method with the best results and the best performance.

The transfer function of a 2-D FIR filter of dimension $\mathrm{N}_{1} \mathrm{XN}_{2}$ is given by $[12,19]$ :

$H\left(Z_{1}, Z_{2}\right)=\sum_{n_{1}=0}^{N_{1}-1} \sum_{n_{2}=0}^{N_{2}-1} h\left(n_{1}, n_{2}\right) * Z_{1}^{-n_{1}} * Z_{2}^{-n_{2}}$

Where $h\left(n_{1}, n_{2}\right)$ is its impulse response.

The frequency response of a 2-D FIR digital filter with its impulse response $h\left(n_{1}, n_{2}\right)$ is given by:

$H\left(\omega_{1}, \omega_{2}\right)=\sum_{n_{1}=0}^{N_{1}-1} \sum_{n_{2}=0}^{N_{2}-1} h\left(n_{1}, n_{2}\right) * e^{-j .\left(n_{1} \omega_{1}+n_{2} \omega_{2}\right)}=M\left(\omega_{1}, \omega_{2}\right) * e^{j . \theta\left(\omega_{1}, \omega_{2}\right)}$

Where $M\left(\omega_{1}, \omega_{2}\right)=\left|H\left(\omega_{1}, \omega_{2}\right)\right|$ and $\theta\left(\omega_{1}, \omega_{2}\right)=\arg \left(H\left(\omega_{1}, \omega_{2}\right)\right)$

$M\left(\omega_{1}, \omega_{2}\right)$, the magnitude response of $H\left(\omega_{1}, \omega_{2}\right)$, is a real-valued function.

Due its symmetrical impulse response, the frequency response is given by: 
$H\left(\omega_{1}, \omega_{2}\right)=M\left(\omega_{1}, \omega_{2}\right) * e^{-j\left[\frac{\left(N_{1}-1\right)}{2} \omega_{1}+\frac{\left(N_{2}-1\right)}{2} \omega_{2}\right]}$

$M\left(\omega_{1}, \omega_{2}\right)=\left|F\left(\omega_{1}, \omega_{2}\right)\right|$

$F\left(\omega_{1}, \omega_{2}\right)=\sum_{k_{1}=0}^{P_{1}} \sum_{k_{2}=0}^{P_{2}} a\left(k_{1}, k_{2}\right) * \cos \left(k_{1} \omega_{1}\right) * \cos \left(k_{2} \omega_{2}\right)$

$P_{1}=\frac{\left(N_{1}-1\right)}{2}$ and $P_{2}=\frac{\left(N_{2}-1\right)}{2}$

Where $\mathrm{N}_{1}$ and $\mathrm{N}_{2}$ are odd integers.

The sum-squared error over these frequency points is given by:

$E=\sum_{j=1}^{m_{1}} \sum_{k=1}^{m_{2}}\left[D\left(\omega_{1 j}, \omega_{2 k}\right)-F\left(\omega_{1 j}, \omega_{2 k}\right)\right]^{2}$

Where $D$ is the desired magnitude response and $F$ the actual magnitude response.

$E_{i}=\sum_{j=1}^{m_{1}} \sum_{k=1}^{m_{2}}\left[D\left(\omega_{1 j}, \omega_{2 k}\right)-C_{1}\left(\omega_{1 j}\right) * A_{i} * C_{2}\left(\omega_{2 k}\right)^{T}\right]^{2}$

Where $A_{i}$ and $E_{i}$ are the $i$-th chromosome and actual sum-squared error obtained with the $i$-th chromosome, respectively.

$C_{1}\left(\omega_{1 j}\right)=\left[\begin{array}{cccc}1 & \cos \left(\omega_{11}\right) & \cdots & \cos \left(P_{1} \cdot \omega_{11}\right) \\ 1 & \cos \left(\omega_{12}\right) & \cdots & \cos \left(P_{1} \cdot \omega_{12}\right) \\ \vdots & \vdots & \vdots & \vdots \\ 1 & \cos \left(\omega_{1 m_{1}}\right) & \ldots & \cos \left(P_{1} \cdot \omega_{1 m_{1}}\right)\end{array}\right]$

$C_{2}\left(\omega_{2 k}\right)=\left[\begin{array}{cccc}1 & \cos \left(\omega_{21}\right) & \cdots & \cos \left(P_{2} \cdot \omega_{21}\right) \\ 1 & \cos \left(\omega_{22}\right) & \cdots & \cos \left(P_{2} \cdot \omega_{22}\right) \\ \vdots & \vdots & \vdots & \vdots \\ 1 & \cos \left(\omega_{2 m_{2}}\right) & \ldots & \cos \left(P_{2} \cdot \omega_{2 m_{2}}\right)\end{array}\right]$

The main idea is to obtain a least-squares approximation to the given magnitude function, which leads to the optimal approximation of the solution.

The matrix $\left(A_{i}\right)$ formed by the filter coefficients of the $i$-th chromosome can be characterized as:

$A_{i}=\left[\begin{array}{cccc}a_{00} & a_{01} & \ldots & a_{0 P_{2}} \\ a_{10} & a_{11} & \cdots & a_{1 P_{2}} \\ \vdots & \vdots & \vdots & \vdots \\ a_{P_{1} 0} & a_{P_{1} 1} & \ldots & a_{P_{1} P_{2}}\end{array}\right]$

$a_{k_{1} k_{2}}$ is $\left(k_{1}, k_{2}\right)^{t h}$ filter coefficient, $k_{1}=0,1, \ldots, P_{1}$ and $k_{2}=0,1, \ldots, P_{2}$.

\section{Proposed new GA for designing 2-D FIR filters}

We propose a new genetic algorithm devoted to FIR filter design that aims to be user-friendly. In order to accelerate the evolutionary process and reach an efficient solution with a reasonable execution time, several mechanisms have been incorporated in this algorithm: firstly the use of an initial population presenting chromosomes close to acceptable solutions coming from recent experiments obtained with standard approaches; secondly, genetic operators, and particularly the mutation operator, that are completely adapted to the level of the genetic process and therefore limit the simultaneous tuning of several static parameters; thirdly, a ranking selection scheme has been introduced in order to limit the promotion of extraordinary chromosomes, thus preventing 
premature convergence. To improve accuracy, the genetic exploration is driven by a fitness assignment strategy including the filter specificities. While driving GAs in this way is necessary, it is however not adaptive enough to continuously manage the trade-off between elitism and pressure preservation. Our algorithm is therefore reinforced by a mechanism involving a refreshing process to reseed the population when necessary. The selection process naturally promotes the best chromosomes. It integrates a function allowing the limitation of redundant chromosomes, hence promoting diversification.

More than existing techniques, like self-adaptation of mutation step-sizes, that can solve this problem partially, our GA allows to regulating selection pressure, mutation and recombination parameters simultaneously. The mechanisms interact with one another and are complementary: some are reccurent as others are activated only in the event of loss of control. They can control and adapt the genetic advances, then put the GA at any time in a configuration in which it can evolve.

Concerning the parameter definition, our GA includes the following complexity: from one side, the contemporary view acknowledges that using the right parameter values can make a big difference in algorithm performance. For the other side, the selected parameter values are not necessarily optimal even if well calibrated. Simultaneous tuning is an almost hopeless task. Any set of static parameters is likely to be inappropriate as different values may work better or worse at different stages of the evolutionary process. One advantage of GAs is that they can perform correctly under a range of parameters. Furthermore, the self-adaptative scheme induces more tolerance in the parameter definition. It is therefore possible to provide starting points (initial parameter calibration) without user intervention (see section 4.5) so that the GA is always correctly driven. This constitutes a real power of our GA and avoids a number of potential pathological issues that can surprise unwary users and discourage them.

\subsection{Main components of the GA}

Genetic algorithm (GA) [32-34] was proposed by Holland as an algorithm for probabilistic search, learning, and optimization. Diversity and elitism are the main factors to be managed with a GA. Population diversity enables the fruitful exploration of the search space $[32,35]$. The main goal of the selection mechanism [36] is to find an efficient balance between the exploration and exploitation abilities of the search during the run.

\section{Chromosome representation:}

The GA is a real-coded genetic algorithm (RCGA) as this representation is more suitable for filter design than binary implementations. Coefficient domains can be large which means that for a convenient fix length for the chromosomes, increasing the domain would limit accuracy. A chromosome solution is then directly represented by a matrix of coefficients representing the filter itself. It means that for a filter of $\mathrm{N}_{1} \mathrm{xN} \mathrm{N}_{2}$ dimensions, it is necessary to determine $\mathrm{N}_{1} \mathrm{XN}_{2}$ coefficients, the goal being to find them in order to minimize the sum-squared error $\mathrm{E}$ over $\mathrm{m}_{1} \mathrm{xm}_{2}$ frequency points. Chromosomes with lower errors will have a higher probability of being selected as parents.

\section{Fitness function:}

The quality of a chromosome solution is given by the fitness value, which is calculated by the fitness function. The fitness value is used by the GA to discriminate between good and not so good designs, so that it can select accordingly. The fitness function is defined as the inverse of the error E presenting the goodness to be maximized. For a given shape of filter, peak ripples are generally more present in certain regions than in others, making their attenuation more difficult. To tackle this situation, we suggest weighting the sum-squared error, which will contribute to compensating this recurrent imbalance. Since the weights are difficult to obtain theoretically due to the lack of advances in GA itself, they have been experimentally obtained for the different filter supports we have managed. The choice of values of the weighting function is given in Table 3, which summarizes the different adjustment parameters of our AGA.

It should be mentioned that there is no interaction with the user.

$E_{i}=\sum_{j=1}^{m_{1}} \sum_{k=1}^{m_{2}} W\left(\omega_{1 j}, \omega_{2 k}\right) *\left[D\left(\omega_{1 j}, \omega_{2 k}\right)-C_{1}\left(\omega_{1 j}\right) * A_{i} * C_{2}\left(\omega_{2 k}\right)^{T}\right]^{2}$ 
Where $W$ is the weight matrix of $\mathrm{m}_{1} \mathrm{xm}_{2}$ dimensions.

$D$ is a matrix of dimensions $m_{1} x_{2}$ which gives the desired magnitude response

$C_{1}$ is the cosine matrix of dimensions $\mathrm{m}_{1} \mathrm{xP}_{1}$

$C_{2}$ is the cosine matrix of dimensions $\mathrm{m}_{2} \mathrm{xP}_{2}$

The matrix $A_{i}$ formed by the filter coefficients of the $i$-th chromosome has dimensions $\mathrm{P}_{1} \mathrm{xP}_{2}$

The fitness evaluation of each chromosome concentrates the cost of the AGA.

The fitness function is calculated for each chromosome in different stages of the adaptive genetic algorithm. To calculate the fitness function of each chromosome, it is necessary to multiply the cosine matrix $C_{1}$ by the matrix coefficient $A_{i}$. The result is multiplied by the transpose of the matrix cosine $C_{2}$. Then one carries out the subtraction between the matrix containing the desired magnitude response and the result of the product matrix. And finally, the fitness value is obtained by multiplying the result of the subtraction and the weight matrix $W$. These operations (evaluation of the fitness function) are performed in three steps of our algorithms, i.e., mutation, crossover and selection.

\section{Initial population:}

The performance of the GA depends on the initial population from which the solution starts to evolve. A chromosome is generated as follows:

$P O P_{0}=\left(\frac{1}{a}\right) * \operatorname{randn}\left(N_{1}, N_{2}\right)$

Where $\mathrm{N}_{1} \mathrm{XN}_{2}$ is the filter size, $a$ is an integer between 5 and 20 .

In Matlab, this instruction generates pseudorandom numbers from a normal distribution with mean 0 and standard deviation $(1 / a)$.

This allows the initial population to have appropriate values compared to our optimization problem (Experiments have shown that the standard deviation between the genes of chromosomes is less than 0.2 ). The way to generate the initial population does not affect the quality of results, but may increase or decrease slightly the time required for convergence.

If available, $P O P_{0}$ can be partially represented by solutions obtained with standard approaches and mutated. While not systematic, this process generally speeds up the genetic convergence (CPU time and iteration number to obtain satisfying solutions).

\section{Genetic strategy:}

The selected scheme is classically driven by the different operators of crossover, mutation, selection and reproduction as illustrated in Fig. 1 and 2. It includes however some major differences detailed later in the way the successive operations are applied.

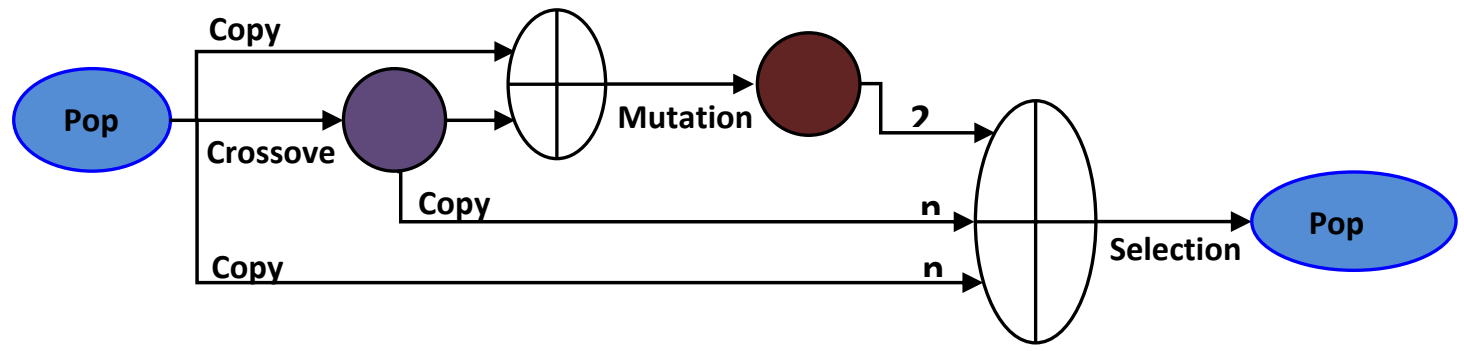

Figure 1. Genetic process.

The $n$ chromosomes from generation $t$ produce $2 * n$ chromosomes via the crossover operator. In this scheme, there is no need to fix a crossover rate. A copy of the parent and child population is kept. Parents and children are put together and submitted to the mutation operator. Each chromosome component has a $P_{M}$ probability of being mutated. The $P_{M}$ value is calculated for each chromosome individually as well as the level of mutation and is dependent on the genetic advance of the algorithm. It is a so-called self-adaptive parameter. An enlarged 
population of $4 * n$ chromosomes is then submitted to the selection operator that discards the redundant and worse chromosomes to obtain a population of size $n$.

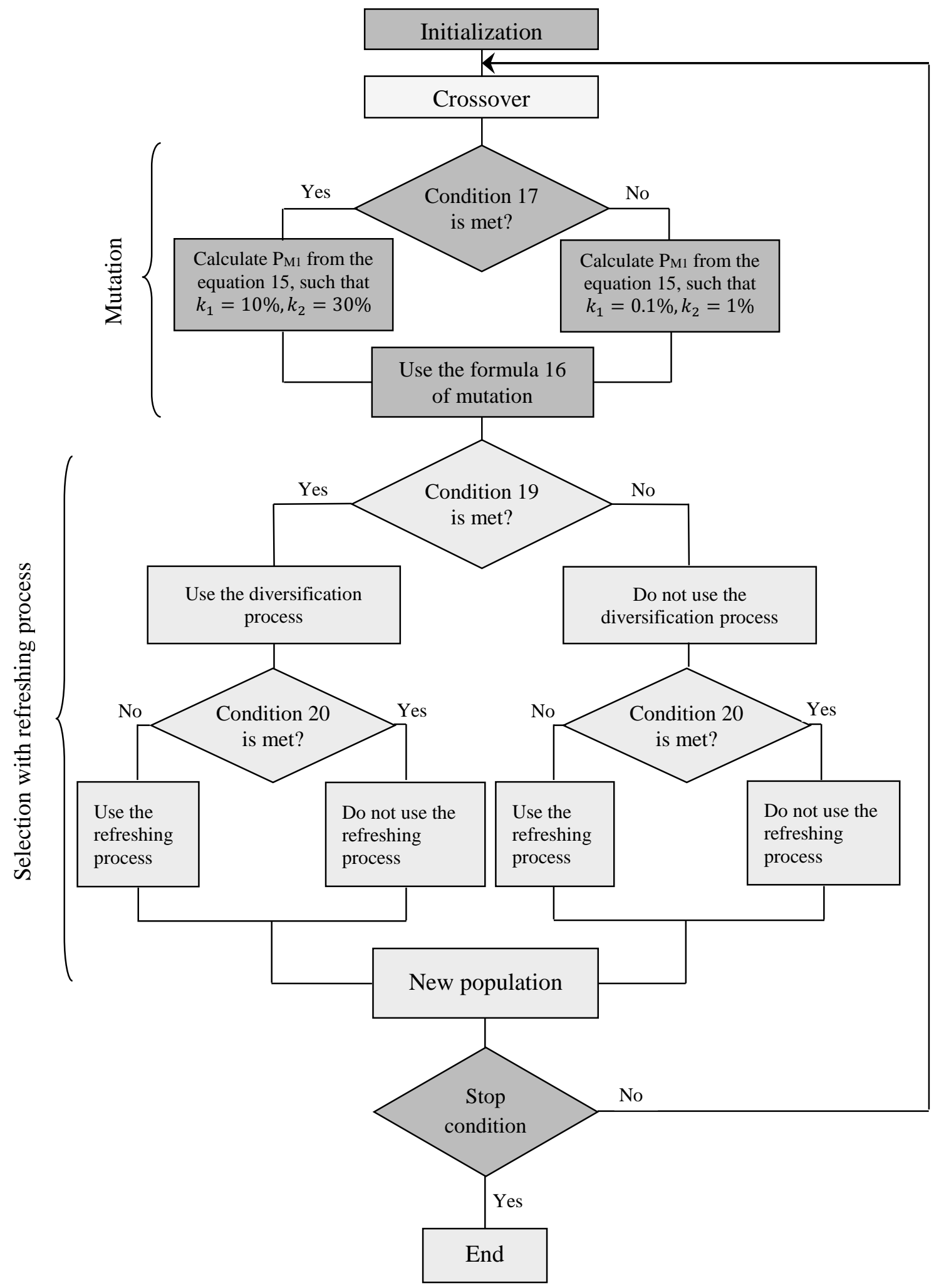

Figure 2. Flowchart of the AGA.

The selection operator takes into account both elitism and diversity. The decision to select a given chromosome in the new population is based on two criteria; the first one is the fitness score. If there exists a 
chromosome in the candidate population (among the $4 * n$ ) with a higher score than the other candidates, it is likely to be selected. This is the elitist side of the process. However, if a candidate fulfills this elitist criterion but presents a comparable structure with a chromosome that has already been selected, it is likely to be rejected. This is the diversity side of the process. Our scheme implicitly includes the well-known crowding and niching concepts [37]. It is very fast and easy to implement as it requires only the definition of similarity between two chromosomes. Although the evaluation is carried out on a population of $4 * n$ individuals and takes longer, the number of iterations is reduced.

When this process generates less than $n$ chromosomes, the population is completed with new chromosomes generated from the last population having being submitted to a large mutation.

\subsection{Genetic operations and dedicated mechanisms to improve convergence}

Some studies have put considerable effort into finding parameter values which give a reasonable performance for a wide range of problems. Specific problems however require dedicated parameters stressing the need for additional mechanisms that allow good parameter tuning methods. Our algorithm hybridizes ingredients from the two approaches. Our crossover scheme is based on a standard support, while the mutation and selection schemes are more sophisticated. They include mechanisms that contribute to GA flexibility and encourage a minimum of diversity among the population.

\section{Crossover operator:}

Crossover is the primary operator in GAs and is the key to their success. Direction-based crossover uses the values of an objective (fitness) function in determining the direction of genetic search.

In this case we do not have to worry about a high crossover rate (equal to 1) because the new population is selected from the best between parents and children.

The operator generates two children $C h_{C}^{\text {Child }}$ from two parents $i$ and $j$ according to the following rule:

$\left\{\begin{array}{l}C h_{C}^{\text {Child } 1}=\left(C h^{i}-C h^{j}\right) * \lambda_{c 1}+C h^{i} \\ C h_{C}^{C h i l d 2}=\left(C h^{j}-C h^{i}\right) * \lambda_{c 2}+C h^{j}\end{array}\right.$

Where $C h^{i}\left(C h^{j}\right)$ presents the individual $i(j)$, and $\lambda_{c 1}$ and $\lambda_{c 2}$ are random numbers between 0 and 1 .

$\begin{cases}\text { If }(\text { fit }(i)>f i t(j)) & \lambda_{c 1}>\lambda_{c 2} \\ \text { Else } & \lambda_{c 1}<\lambda_{c 2}\end{cases}$

Crossover enables the generation of new chromosomes without introducing any new genetic features into the population at the gene level. Since genes can only reproduce or die, if at a certain position all the genes have the same value, there is no way that crossover can recover the lost genetic feature.

\section{Mutation operator:}

The Mutation operator arbitrarily alters the gene value according to a predetermined probability. The mutation operator makes a significant contribution to the search effectiveness. It introduces diversity and reflects features which are not present in the current population, and therefore can protect against such a harmful loss and prevent premature convergence. The mutation strategy is recognized as one of the GA components that has the greatest influence on performance. Classically, the mutation operator is applied for randomly chosen individuals, the number of them varying with the so called mutation probability. Compared to the traditional mutation operator we suggest a non-uniform mutation directly applicable to each chromosome of the population; the non-uniformity is related to the chromosomes fitness. In order to contribute efficiently to a better diversity, we also propose an adaptive version that is able to reinforce the mutation action when necessary.

The idea of non-uniform mutation is relatively well-known in the genetic community. The mutation rate adaptively varies according to the average improvement of the best fitness values during a specified number of generations. The mutation rate is limited for the best chromosomes and encouraged for the worst. For a given chromosome $i$, $P_{M_{i}}$ is applied to each gene. 
$P_{M_{i}}=\left\{\begin{array}{cc}\frac{k_{1} *\left(f_{\max }-f_{i}\right)}{\left(f_{\max }-f_{\text {avg }}\right)} & f_{i} \geq f_{\text {avg }} \\ k_{2} & f_{i}<f_{\text {avg }}\end{array}\right.$

Where $f_{\max }$ and $f_{\text {avg }}$ are the maximum and average fitness values in the current population, respectively, and $f_{i}$ is the fitness value of the current chromosome $i . k_{1}$ and $k_{2}$ are weighting parameters with $k_{1}$ and $k_{2} \leq 1$. They are respectively the lower bound and upper bound of the weighting parameters of the mutation rate.

As explained above, this adaptation concerns the differential between the fitness values of the chromosomes. This dynamic is to a certain extent dissociated from the genetic advance which can leave the practitioners without any help for a given situation.

Confidence intervals for good values for the weighting parameters $k_{1}$ and $k_{2}$ were determined so as to be available before running the algorithm. The values were evaluated only on a limited scale and the perfect configuration does not exist. The parameter performance landscape changes over time as it is dependent on the genetic advances. Tuning these parameters is fruitless and therefore not necessary. The novelty of our approach resides in the conditions of use by distinguishing the state where the population evolves in a normal manner and from a state where the population is diverse.

The simple idea consists in restarting an evolution process that has stalled by refreshing more chromosomes. The mutation rate $P_{M_{i}}$ is increased once via the $k_{1}$ and $k_{2}$ values. It is actually increased each time the population tends to get stuck in a local optimum and decreased when the population is scattered in the search space. The probability of mutation $P_{M_{i}}$ is applied to each gene as explained. If the mutation is activated, its level of mutation also takes feedback from the current state of the search and modifies the chromosomes accordingly.

Different approaches can be investigated. We suggest adopting a two-case version described as follows:

$C h^{\text {new }}=\left\{\begin{array}{cc}C h^{\text {old }} *\left(1+\lambda_{m 1}\right) & \text { if local minimum } \\ C h^{\text {old }} *\left(1+\lambda_{m 2}\right) & \text { else }\end{array}\right.$

The operator generates new chromosomes $C h^{\text {new }}$ from old chromosomes $C h^{\text {old }}$.

Where $\lambda_{m 1}, \lambda_{m 2}$ are parameters depending on the genetic state $\left(\lambda_{m 1} \gg \lambda_{m 2}\right)$ and $C h$ defines each chromosome component.

The presence of a local minimum is estimated if three criteria are simultaneously activated:

- A sufficient advance of genetic life characterized by the average level of fitness function of candidate solutions that is compared to a threshold $t \mathrm{~h}_{1}\left(f_{\text {avg }} \leq t \mathrm{~h}_{1}\right)$.

- Absence of a population change between two genetic iterations characterized by the value of the derivative of the average fitness function $\left(\Delta_{\text {fitness(avg) }} \leq t \mathrm{~h}_{2}\right.$ where $t \mathrm{~h}_{2}$ is a threshold).

- Absence of a gap between the best genetic solution and the average of the solutions $\left(\frac{f_{\max }-f_{\text {avg }}}{f_{\text {avg }}} \leq t h_{3}\right.$, where $f_{\max }$ and $f_{\text {avg }}$ are respectively the best and average fitness solutions and $t \mathrm{~h}_{3}$ a threshold).

When this mechanism is active in our algorithm, we say that an adaptive non-uniform mutation is applied.

The process of verification of the presence of a local minimum can be summarized in this equation:

$$
\left\{\begin{array}{l}
\text { If }\left(f_{\text {avg }} \leq t \mathrm{~h}_{1} \text { and } \Delta_{\text {fitness }(\text { avg })} \leq t \mathrm{~h}_{2} \text { and } \frac{f_{\text {max }}-f_{\text {avg }}}{f_{\text {avg }}} \leq t h_{3}\right) \text { Presence of a local minimum } \\
\text { Else No presence of a local minimum }
\end{array}\right.
$$

The detection of a local minimum is based only on the fitness values. It presents a very low computational cost as it is based on simple calculations. An elementary calibration procedure makes it possible to find confidence intervals. 


\section{Selection scheme refinement}

The strategy for parent selection has a great impact on GA performances. Several researches think that manipulating parameters regarding the selection mechanisms and the population can be more rewarding than tuning crossover and mutation rates. The implemented mechanism is a self-adaptive scheme based on a process where the parameters are implicit, i.e. they are selected by the evolutionary cycle itself. They not require any intervention of the user before the run or any tuning. The balance between selection pressure and diversification is achieved through the strategy and not through parameters that need to be changed during the evolutionary process. The strategy has been largely validated for the filter design problem.

We used selection based on an enlarged sampling space; both parents and offspring have the same chance of competing for survival.

Our selection scheme aims at improving the quality of the current population by giving chromosomes of higher quality a higher probability to be inserted into the next population without reducing the population diversity. Then, the selection scheme allows the GA dealing with multimodal functions and can explore several hills in the fitness landscape simultaneously.

$P_{i}=f_{i} / \sum_{j=1}^{n} f_{j}$

Where $f_{j}$ is the fitness value of the $j^{\text {th }}$ chromosome.

The scheme is not a pure ranking process but acts with the same objective, i.e. preventing very fit individuals from gaining dominance early at the expense of less fit ones.

The ranking is based purely on the probability differences but the selection algorithm includes the genetic material.

The algorithm starts with the best chromosome called current. This chromosome is automatically selected and first compared to the second called inspected via the fitness function. If there is no fit between the two, the second becomes current and the procedure continues. Otherwise, the comparison is done via a gene metric to avoid discarding two chromosomes having similar fitness values while being genetically different. Inspected is discarded if there is no fit between them in this space. In this case the first chromosome is still current. The comparison is then done with the third chromosome which becomes inspected. The procedure continues until the whole population has been inspected.

This mechanism contributes to a better diversification and is therefore called "diversification".

$\left\{\begin{array}{l}\text { if } \Delta_{\text {fitness }}\left(\text { ch }_{\text {current }}, c h_{\text {inspected }}\right) \geq f_{\varepsilon} \text { continue else } \\ \text { if } \Delta_{\text {gene }}\left(c_{\text {current }}, c h_{\text {inspected }}\right) \geq g_{\varepsilon} \text { continue else } \\ \text { discard } c h_{\text {inspected }}\end{array}\right.$

ch $h_{\text {current }}\left(c h_{\text {inspected }}\right)$ stands for chromosome current as $\Delta_{\text {fitness }}\left(r e s p . \Delta_{\text {gene }}\right)$ stands for distance in the fitness space, and $f_{\varepsilon}$ (resp. $g_{\varepsilon}$ ) is a predefined threshold.

The cost of the selection scheme is related to the ranking in the fitness and gene space; $n-1$ distances have to be calculated in each space. Concerning the gene space, different distances can be selected: direct Euclidean distances in subspaces of selected filter coefficients as a difference between a function of filter coefficients like a simple sum. The second is preferable as the cost is heavy for the first. We begin by comparing the genes in positions $(1,1),\left(1, P_{2}\right),\left(P_{1}, 1\right)$ and $\left(P_{1}, P_{2}\right)$ of the individual previously selected and the individual inspected. Then, we randomly draw others to compare positions. In general, the first four comparisons are sufficient to confirm or deny a similarity between genes of two chromosomes.

The left element of the position is the line index of the gene given by the matrix $A_{i}$ and the right element of the position is the column index of the given gene by the matrix $A_{i}$.

It should be noted that the second calculation is processed only when current and inspected fit very well in the fitness space. This speeds up the process. New promising chromosomes in the population are inserted by replacing the most similar ones in the spirit of a crowding scheme. This mechanism is called adaptive selection as it induces 
automatic modification of the selection probability. The fitter individuals are selected and at the same time the population diversity is maintained.

\subsection{Refreshing process to prevent premature convergence}

Equilibrium between selective pressure and population diversity depends on the problem to be solved. Unfortunately, there exists no manageable model for a controllable selection pressure. As already mentioned, our strategy consists in defining parameters that give a reasonable performance for a wide range of problems while affording our algorithm some flexibility and adaptation. For these reasons, the flexibility embedded in our mutation operator is an interesting feature but it cannot prevent and manage all the cases of premature convergence. The same holds for the selection scheme.

We therefore introduce the notion of population refreshing (breaking) which involves a profound change in the current population.

Before providing a convenient solution, premature convergence has to be detected. The detection consists in identifying an evolution process that has stalled, materialized by the presence of many similar chromosomes within the population. According to a given metric, one way aims at calculating or estimating the percentage of similar chromosomes within the population and reseeding the population accordingly. Two chromosomes are said to be similar if their distance is less than a predefined threshold depending on the active metric. Although efficient, this approach can be time-consuming. For $n$ chromosomes, this process requires the calculation of $n *(n-1) / 2$ distances on the gene space.

A quicker way consists in working directly on the fitness space and applying the following rule: the current population needs to be reseeded when the difference between the fitness function of the best chromosome and the average fitness function of the population is below a given threshold. In both cases, reseeding is submitted to the level of the genetic advance if this information is available. This is usually the case.

$\left\{\begin{array}{l}\text { if } \Delta_{\text {fitness }}\left(f\left(\text { ch }_{\text {best }}\right), f\left(\text { ch }_{\text {ave }}\right)\right) \leq \Delta f_{\text {min }} \quad \text { and } \\ f\left(\mathrm{ch}_{\text {best }}\right) \leq f_{\text {end }} \text { refreshing process active else } \\ \text { refreshing process inactive }\end{array}\right.$

Where $\Delta f_{\text {min }}$ and $f_{\text {end }}$ are the different thresholds and $f(x)$ the fitness of chromosome $x$.

We suggest a refreshing mechanism (or breaking process) capable of reseeding the active population without losing (Eq. 20) its current advance.

The refreshing is simply a stronger mutation (Eq. 16) characterized by a higher probability and higher rate. This mechanism is highly optimized and acts in complementarity to the other ones i.e. adaptive mutation and diversification procedure. In this case, the mutation probability is fixed at a high value such as $P_{M}=40 \%$.

\section{Experimental results}

In this section we aim at experimentally demonstrating the effectiveness of our approach when applied on various cases. Different versions of our AGA (Adaptive Genetic Algorithm) approach are evaluated to point out the role of the different mechanisms introduced. $\mathrm{GA}_{1}$ includes all the new mechanisms embedded in our initial version $\mathrm{GA}_{4}$ that stands more for a standard GA. The components of each version are detailed in Table 1.

Comparisons are made with other algorithms. These algorithms include well-known conventional approaches as well as evolutionary ones. Among the evolutionary approaches, four non-genetic approaches are used in their initial versions; the parameters are listed in Table 2. Among the conventional approaches, two of them based on Remez and least squares were tested. For both methods, the 1-D filter was synthesized and then we used the frequency transformation to design the 2-D filter. Particular attention was paid to two popular genetic approaches often cited in recent papers and specifically developed for filter design. 
Table 1: Description and parameters of proposed GA versions

\begin{tabular}{|c|c|c|c|}
\hline & \multicolumn{2}{|l|}{ Parameters } \\
\hline \multirow{4}{*}{ 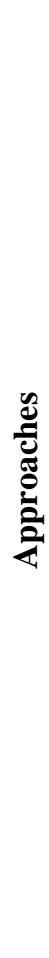 } & Adaptive $\mathbf{G A}_{1}$ & $\begin{array}{l}\text { - } \\
\text { - diversification procedure } \\
\text { - } \quad \text { Presence of a local minimum } \\
\text { - } \quad \text { Refreshing process active } \\
\text { - } \quad \text { Direction-based crossover } \\
\text { - } \quad \text { Adaptive selection with the refreshing procedure } \\
\text { (diversification and breaking process) }\end{array}$ & $\begin{array}{l}k_{1}=0.1 \%, k_{2}=1 \% \\
k_{1}=10 \%, k_{2}=30 \% \\
P_{M}=40 \% \\
C_{R}=100 \%\end{array}$ \\
\hline & Adaptive $\mathbf{G A}_{2}$ & $\begin{array}{l}\text { - } \quad \text { Non-uniform mutation } \\
\text { - } \quad \text { Refreshing process active } \\
\text { - } \quad \text { Direction-based crossover } \\
\text { - } \quad \text { Adaptive selection with the refreshing procedure } \\
\text { (diversification and breaking process) }\end{array}$ & $\begin{array}{l}P_{M}=0.5 \% \\
P_{M}=40 \% \\
C_{R}=100 \%\end{array}$ \\
\hline & Adaptive $\mathbf{G A}_{3}$ & 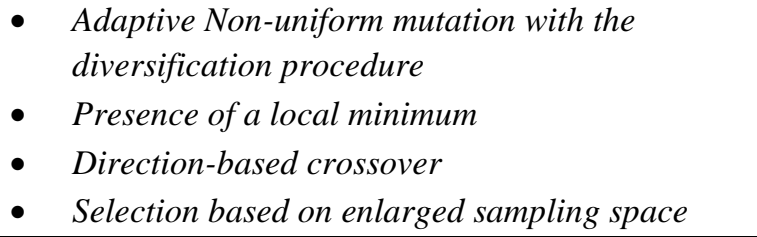 & $\begin{array}{l}k_{1}=0.1 \%, k_{2}=1 \% \\
k_{1}=10 \%, k_{2}=30 \% \\
C_{R}=100 \%\end{array}$ \\
\hline & $\begin{array}{l}\text { Standard GA4 } \\
{[18]}\end{array}$ & $\begin{array}{ll}\text { - } & \text { Non-uniform mutation } \\
\text { - } & \text { Direction-based crossover } \\
\text { - } & \text { Selection based on enlarged sampling space }\end{array}$ & $\begin{array}{l}P_{M}=0.5 \% \\
C_{R}=100 \%\end{array}$ \\
\hline
\end{tabular}

Table 2: Parameters of each evolutionary approach [17]

\begin{tabular}{|c|c|c|c|c|c|}
\hline & \multicolumn{4}{|c|}{ Parameters } \\
\hline \multirow{3}{*}{ : } & $\begin{array}{l}\text { Simulated } \\
\text { Annealing } \\
{[17]}\end{array}$ & $\begin{array}{l}\text { Random choice of } \\
\text { an initial solution } \\
x_{0} \text { in } S .\end{array}$ & $\begin{array}{l}\text { initial and final } \\
\text { temperature: } \\
T_{0}=2000 T_{F}= \\
10^{-5}\end{array}$ & $k_{B}=1$ & $\begin{array}{l}\operatorname{Pr}(\text { accepted }) \\
=\exp \left(-\Delta E / k_{B} \cdot T\right)\end{array}$ \\
\hline & $\begin{array}{l}\text { Tabu } \\
\text { search [17] }\end{array}$ & $\begin{array}{l}\text { Random choice of } \\
\text { an initial solution } \\
x_{0} \text { in } S .\end{array}$ & $\begin{array}{l}\text { Initial tabu list : } \\
T=\varnothing\end{array}$ & $\begin{array}{l}\text { Size of tabu list } T: \\
S T L=80\end{array}$ & $\begin{array}{l}\text { Size of subset: } S S= \\
100\end{array}$ \\
\hline & $\begin{array}{l}\text { Hill- } \\
\text { climbing } \\
{[17]}\end{array}$ & $\begin{array}{l}\text { Random choice of } \\
\text { an initial solution } \\
x_{0} \text { in } S .\end{array}$ & $k=n_{1} * n_{2}$ & & $\begin{array}{l}\text { Size of subset: } S S= \\
100\end{array}$ \\
\hline
\end{tabular}

Finally, the set of selected algorithms is:

1. $\mathrm{GA}_{1}$

2. $\mathrm{GA}_{2}$

3. $\mathrm{GA}_{3}$

4. $\mathrm{GA}_{4}[18]$

5. Simulated Annealing [17]

6. Tabu Search [17]

7. Hill Climbing [17]

8. Remez and Least Square methods (Frequency Transformation)

9. Frequency Sampling

10. Windowing Method

11. Mastorakis et al. real [31] (real coded version)

12. Mastorakis et al. bin [31] (binary version identical to the initial study)

13. Tzeng (Shian-Tzan Tzeng approach) [13] 


\subsection{Preliminary experiments}

\section{Design of symmetric low-pass 2-D FIR digital filters based on a given amplitude response}

In this paper, we will take full advantage of the minimization of least-squares approximation to deal with the design of 2-D linear-phase FIR digital filters with symmetric properties of 2-D sequences. To illustrate the proposed techniques, we first consider the design of a low-pass 2-D FIR filter for which ten independent runs of each algorithm are performed. It should be remembered that the filter coefficients in GA are represented as genes in an individual. Simulations were performed using a computer based on an Intel Core I7 system with Matlab 7.8.0 (R2009a) software package.

This example deals with the design of a filter with the desired magnitude response

$D\left(\omega_{1}, \omega_{2}\right)= \begin{cases}1 & \left|\omega_{1,2}\right|<0.5 \\ 0 & \left|\omega_{1,2}\right| \geq 0.5\end{cases}$

The frequency response of a symmetric 2-D FIR digital filter with dimension N1xN2 can be characterized by Eqs. (2), (3), (4) and (5). $F\left(\omega_{1}, \omega_{2}\right)$ (Eq. 5) is the magnitude response which is used to approximate the desired magnitude response $D\left(\omega_{1}, \omega_{2}\right)$ in the least-squares error sense. That is to say, we want to minimize the error function defined in Eq. (11).

Here, a 7x7 symmetric low-pass digital 2-D FIR filter is designed. The resulting magnitude responses are shown in Fig. 3, 4 and 5. In the first simulation, we tested $\mathrm{GA}_{1}$ by varying some input parameters in order to judge the GA flexibility (Table 3).

Table 3: Parameter setting of the AGA

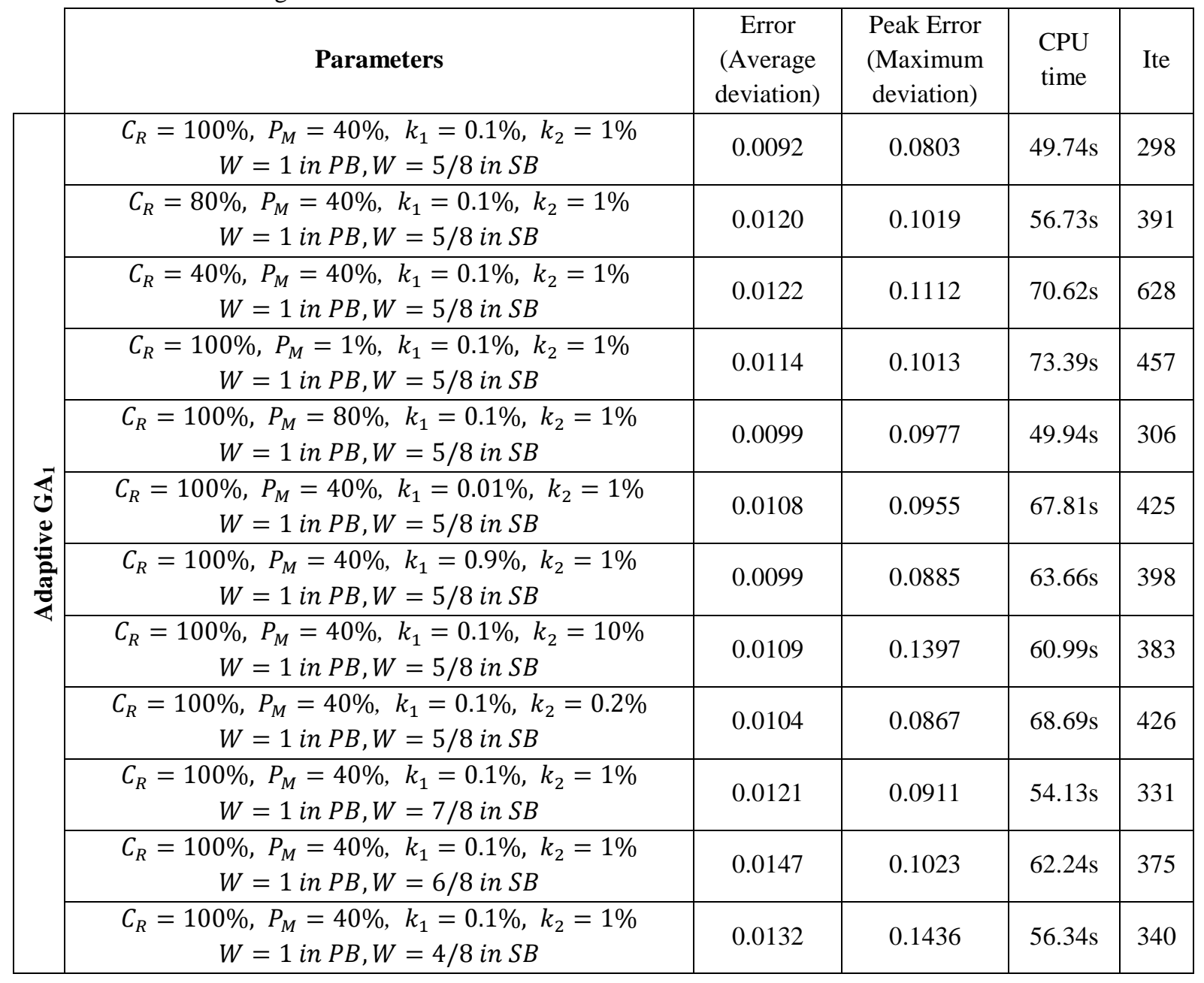




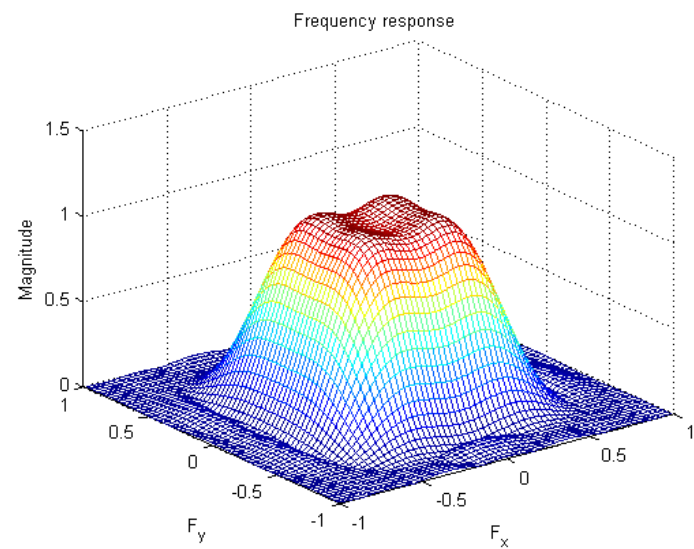

(a)

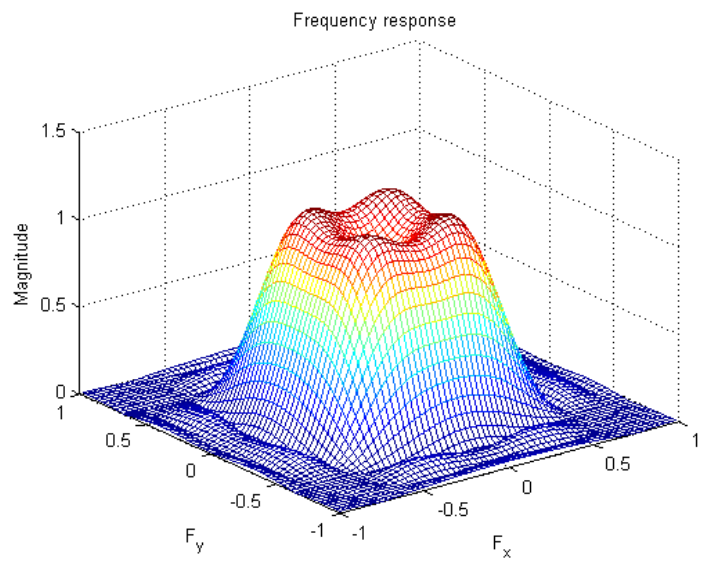

(c)

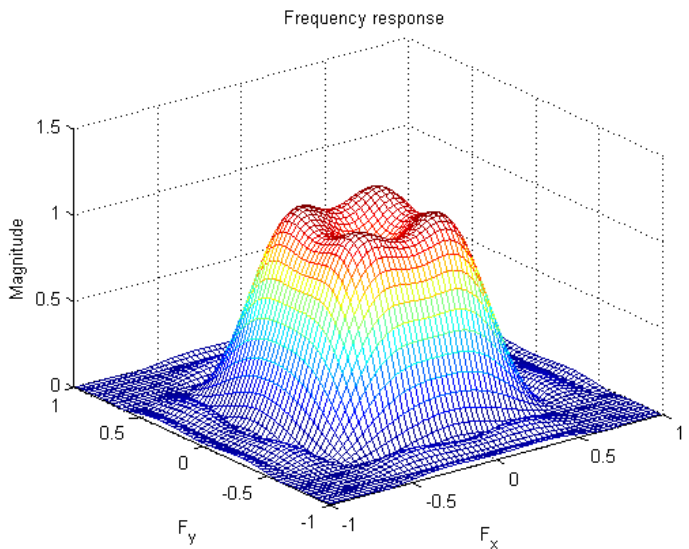

(b)

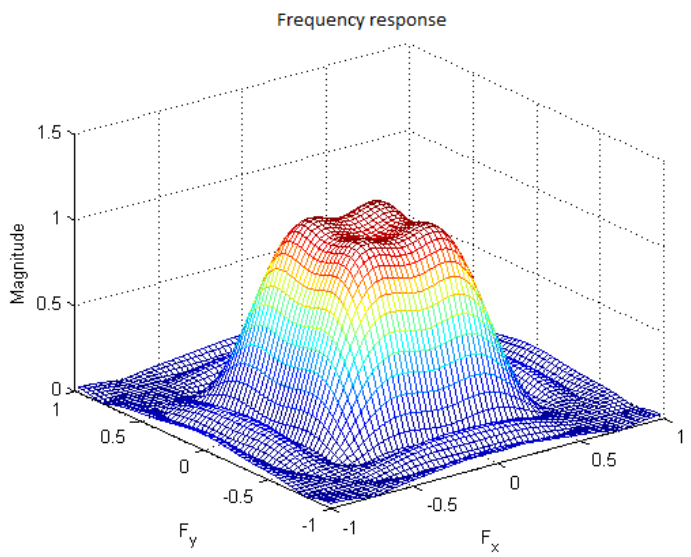

(d)

Figure 3. Frequency responses: (a) Adaptive $\mathrm{GA}_{1}$ (b) Adaptive $\mathrm{GA}_{2}$ (c) Adaptive $\mathrm{GA}_{3}$ (d) Standard $\mathrm{GA}_{4}$. 


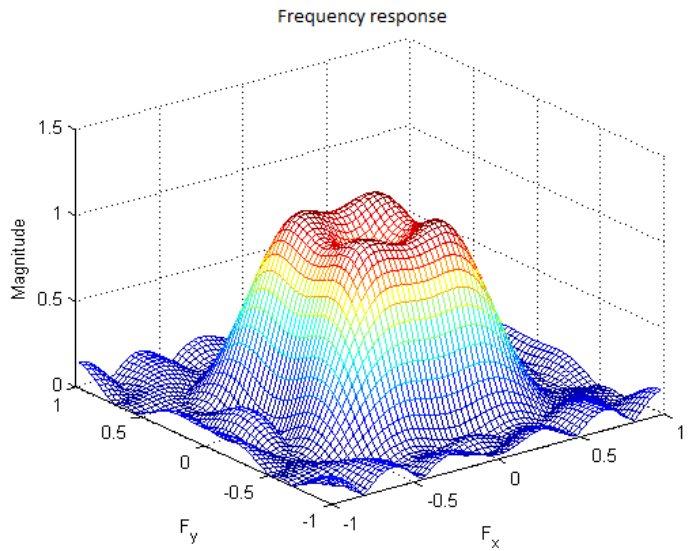

(a)

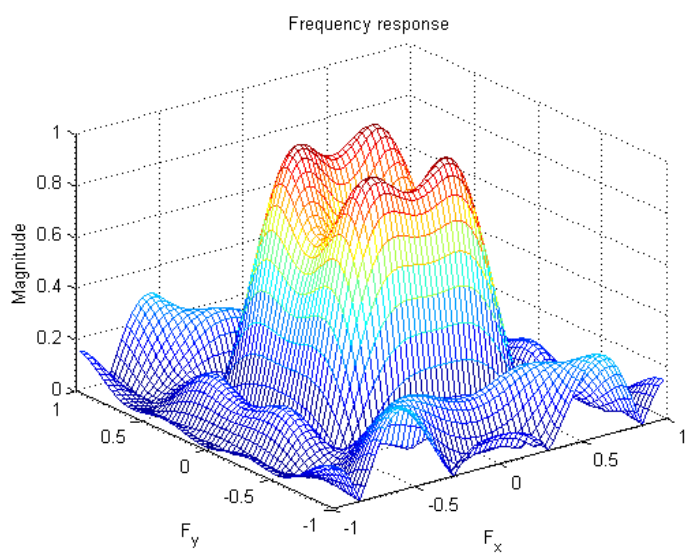

(c)

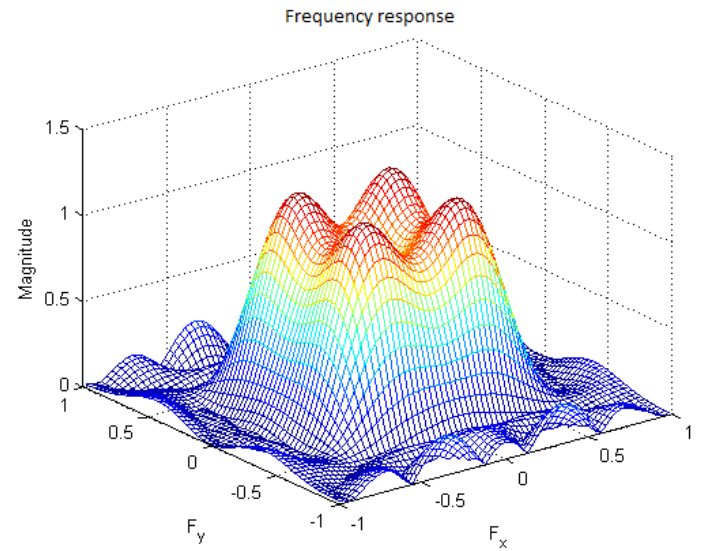

(b)

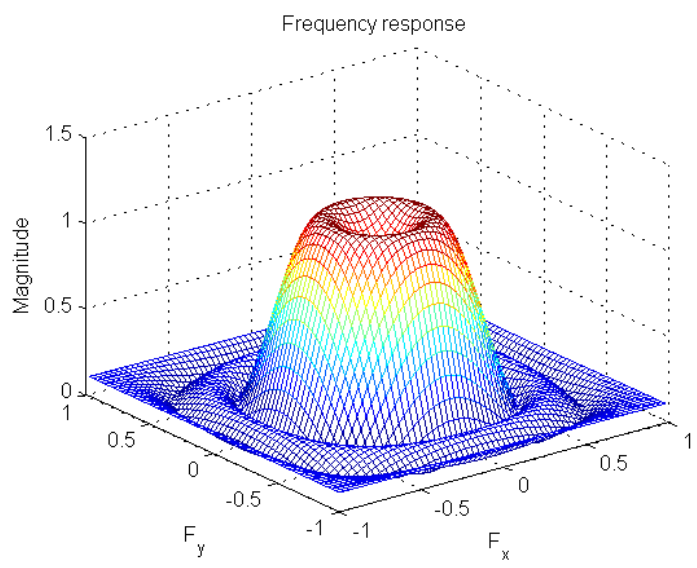

(d)

Figure 4. Frequency responses: (a) Simulated annealing approach (b) Tabu search approach (c) Hillclimbing approach (d) Remez method (frequency transformation). 


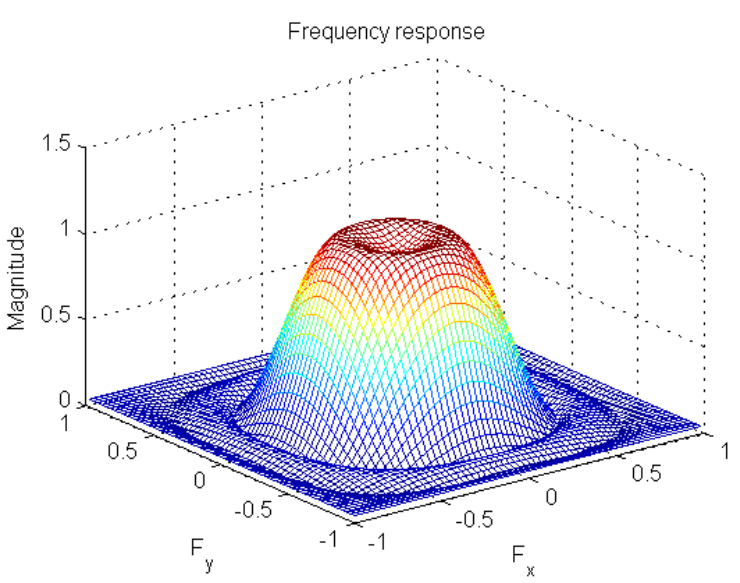

(a)

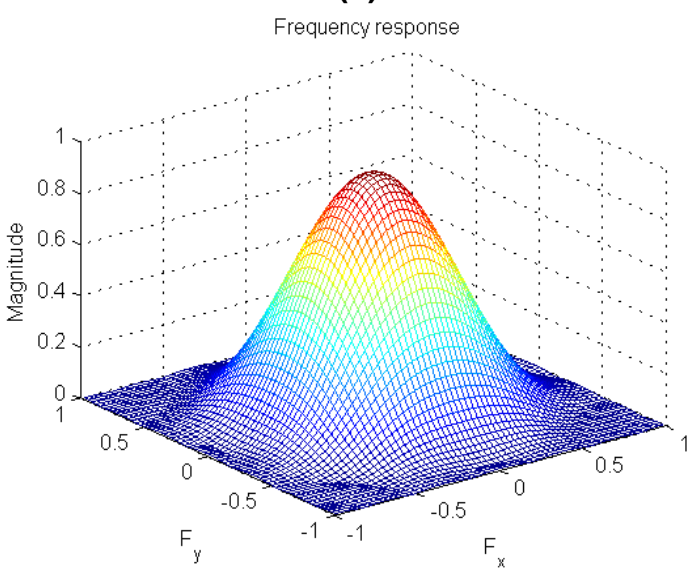

(c)

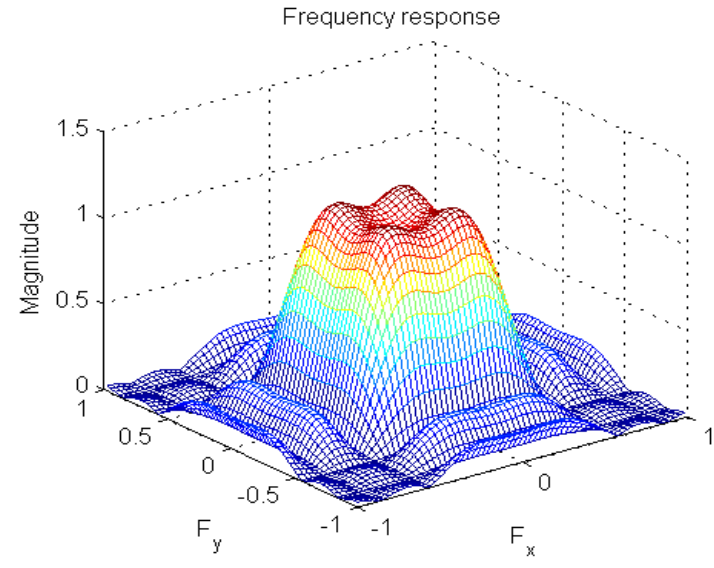

(b)

Figure 5. Frequency responses: (a) Least square method (Frequency transformation) (b) Frequency sampling method (c) Windowing method.

The main figures of our GA (from $\mathrm{GA}_{4}$ to $\mathrm{GA}_{1}$ ) are depicted in Table 1. The first line summarizes the parameter settings of our AGA in its complete version $\left(G_{A}\right)$ for the synthesis of FIR filters. $C_{R}$ and $P_{M}$ respectively stand for the basic crossover and mutation probability defined in section 3.2.

The parameters $f_{\varepsilon}, g_{\varepsilon}$, and $f_{\text {end }}$ were respectively fixed at $0.00001,0.0001$, and $0.008 . \Delta f_{\min }=0.07 * f i t_{\text {ave }}$, $f i t_{\text {ave }}$ being the fitness average of the whole population. The parameters $k_{1}$ and $k_{2}$ were respectively fixed at 0.001 and 0.01 for the normal genetic advance and increased to 0.1 and 0.3 when a sign of premature convergence was detected.

The parameters $t \mathrm{~h}_{1}, t \mathrm{~h}_{2}$ and $t \mathrm{~h}_{3}$ were respectively fixed for all trials at $0.01,0.00001$ and 0.07 .

The parameters $\lambda_{m 1}$ and $\lambda_{m 2}$ were set at $0.5 *$ rand $n$ and $0.05 *$ randn, where randn stands for a random number between 0 and 1 . If the refreshing process was active, the mutation probability was set at $P_{M}=40 \%$.

For a fair comparison, we consider only initial chromosomes that were randomly generated, since CPU time is generally reduced by $0 \%$ to $30 \%$ when initial chromosomes are composed by other solutions from conventional approaches (mutated or not).

In the GA and AGA, the population size $P_{S}$ was fixed at 100, a maximum number of genetic generations was set at 1500 and an implicit probability of crossover $\left(C_{R}\right)$ at $100 \%$.

The weight matrix $W$ is composed of two values: $C_{0}=1$ in the passband and $C_{1}=5 / 8$ outside.

Fig. 3, 4 and 5 show the frequency response obtained for a low-pass filter of $7 \times 7$ dimensions for the different evolutionary algorithms introduced in the previous sections.

The average of the results obtained by each algorithm in all the data sets evaluated is shown in Table 4, which summarizes the performance characterized by five criteria: the average deviation, Peak Error, width of transition band, CPU time and the iteration number. It can be seen that the results are satisfactory but more importantly, that the variations among the configurations are relatively weak. 
The errors of the proposed AGAs are significantly lower than those obtained when other techniques are used. The peak errors of our $\mathrm{GA}_{1}$ approach and standard GA and SA are respectively 0.0803 and 0.1086 and 0.1320 .

Table 4: Parameters of performance obtained for a low-pass filter

\begin{tabular}{|c|c|c|c|c|c|}
\cline { 2 - 5 } \multicolumn{1}{c|}{} & $\begin{array}{c}\text { Error } \\
\text { (Average } \\
\text { deviation) }\end{array}$ & $\begin{array}{c}\text { Peak Error } \\
\text { (Maximum } \\
\text { deviation) }\end{array}$ & $\begin{array}{c}\text { width of } \\
\text { transition band }\end{array}$ & CPU time & Ite \\
\hline $\mathrm{GA}_{1}$ & 0.0092 & 0.0803 & 0.113 & $49.74 \mathrm{~s}$ & 298 \\
\hline $\mathrm{GA}_{2}$ & 0.0200 & 0.1055 & 0.166 & $75.63 \mathrm{~s}$ & 458 \\
\hline $\mathrm{GA}_{3}$ & 0.0193 & 0.1092 & 0.142 & $59.62 \mathrm{~s}$ & 359 \\
\hline $\mathrm{GA}_{4}[18]$ & 0.0212 & 0.1086 & 0.170 & $205.42 \mathrm{~s}$ & 1380 \\
\hline Simulated Annealing [17] & 0.0255 & 0.1320 & 0.174 & $108 \mathrm{~s}$ & \\
\hline Tabu Search [17] & 0.0570 & 0.2190 & 0.139 & $660 \mathrm{~s}$ & \\
\hline Hill Climbing [17] & 0.0810 & 0.3850 & 0.121 & Random & \\
\hline $\begin{array}{c}\text { Remez (Frequency } \\
\text { Transformation) }\end{array}$ & 0.0566 & 0.1668 & 0.163 & $<\mathrm{s}$ & \\
\hline $\begin{array}{c}\text { Least Square (Frequency } \\
\text { Transformation) }\end{array}$ & 0.0424 & 0.1412 & 0.193 & $<2 \mathrm{~s}$ & \\
\hline Frequency Sampling & 0.0551 & 0.2203 & 0.115 & $<\mathrm{s}$ & \\
\hline Windowing Method & 0.0400 & 0.4920 & 0.322 & $<\mathrm{s}$ & \\
\hline
\end{tabular}

We can observe specifically in Fig. 3 and 4 that adaptive GAs and standard GA have the best magnitude response for the passband and stopband regions. The adaptive GAs have however sharper transition band responses compared to the filter designed by the SA and windowing methods. For the stopband region, the HC algorithm and frequency sampling method produce a filter whose response is a little worse than the others.

We clearly see that $\mathrm{GA}_{1}$ produces better results than the other versions for almost all attributes and that $\mathrm{HC}$ is less relevant than all the others. According to the "error" attribute directly linked to the fitness function, $\mathrm{GA}_{1}$ is the most efficient. We should point out the particular role of the couple "adaptive mutation rate" and "refreshing process". From two errors [0.0092; 0.0803] obtained with $\mathrm{GA}_{1}$, we go to [0.0193; 0.1092] for $\mathrm{GA}_{3}$. For GA 1 , we can observe than both the performance and CPU time are improved. Only a small number of genetic iterations (298) is needed to reach the smallest errors ( 0.0092 for the average error and 0.0803 for the peak error).

Fig. 6 show the effect of the couple "refreshing process" and "adaptive mutation". We can observe a series of successive flat levels. Each time a sign of premature convergence is detected, the population is reseeded, which affects the fitness average without losing the best chromosome. This speeds up the genetic advances and improves the final convergence. 


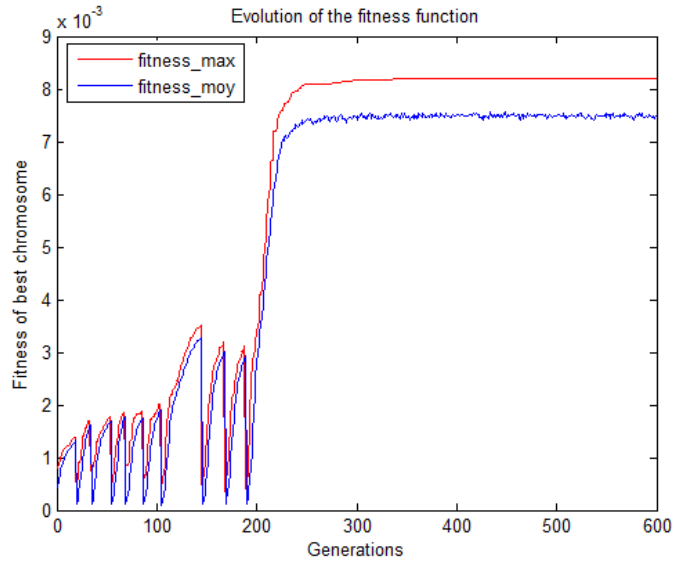

(a)

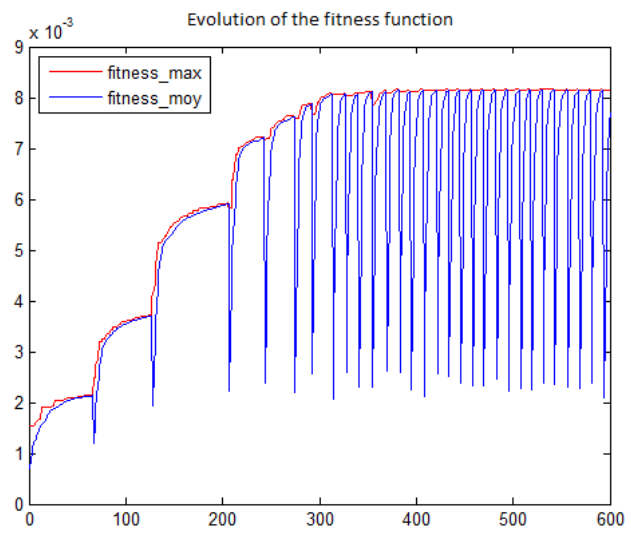

(b)

Figure 6. Effect of the refreshing and breaking process: (a) Refreshing process

(b) Diversification process.

It can be seen that there is a difference between selection with the refreshing process and selection with the diversification process. In the former, the refreshing process occurs almost instantaneously once the algorithm no longer evolves, whereas in the second the diversification process (in order to diversify the population) occurs when certain conditions are met. In the first case, the refreshing process avoids stagnation of the algorithm (the population is varied at any time). Note that the diversification selection does not prevent the breaking process but shortens the population reseeding as the population is more diverse in nature. The diversification process must be followed by the breaking process for the algorithm to give better results. $\mathrm{GA}_{1}$ is better in most of the attributes, underlining its interesting role. We also tested whether the initial population was generated by adding a low noise to the solutions calculated by conventional methods. The GA evolution is different but the final results are similar to those obtained by a pseudo-random initial population.

\subsection{Experiments with other filters}

Table 5 shows a comparative study and performance between the various techniques mentioned in this article applied to the synthesis of a 7x7 symmetric high-pass digital 2-D FIR filter.

As in the case of a low-pass filter, it can be seen that the $\mathrm{GA}_{1}$ (table 5 and Fig. 7.a) is better than other methods for almost all criteria except for the CPU time required: the synthesis of classical algorithms is characterized by a very low CPU time.

Table 5: Parameters of performance obtained for a high-pass filter

\begin{tabular}{|c|c|c|c|c|c|}
\cline { 2 - 5 } \multicolumn{1}{c|}{} & $\begin{array}{c}\text { Error } \\
\text { (Average } \\
\text { deviation) }\end{array}$ & $\begin{array}{c}\text { Peak Error } \\
\text { (Maximum } \\
\text { deviation) }\end{array}$ & $\begin{array}{c}\text { width of } \\
\text { transition band }\end{array}$ & CPU time & Ite \\
\hline $\mathrm{GA}_{1}$ & 0.0108 & 0.0879 & 0.108 & $90.27 \mathrm{~s}$ & 539 \\
\hline $\mathrm{GA}_{2}$ & 0.0199 & 0.1460 & 0.169 & $101.66 \mathrm{~s}$ & 623 \\
\hline $\mathrm{GA}_{3}$ & 0.0133 & 0.1096 & 0.121 & $143.58 \mathrm{~s}$ & 864 \\
\hline GA4 [18] & 0.0182 & 0.1397 & 0.137 & $158.29 \mathrm{~s}$ & 982 \\
\hline Simulated Annealing [17] & 0.0343 & 0.1867 & 0.176 & $104.64 \mathrm{~s}$ & \\
\hline Tabu Search [17] & 0.0855 & 0.2045 & 0.135 & $352.13 \mathrm{~s}$ & \\
\hline Hill Climbing [17] & 0.2512 & 0.9803 & 0.130 & Random & \\
\hline $\begin{array}{c}\text { Remez (Frequency } \\
\text { Transformation) }\end{array}$ & 0.0250 & 0.1026 & 0.166 & $<2 \mathrm{~s}$ & \\
\hline $\begin{array}{c}\text { Least Square (Frequency } \\
\text { Transformation) }\end{array}$ & 0.0208 & 0.1002 & 0.191 & $<\mathrm{s}$ & \\
\hline Frequency Sampling & 0.0352 & 0.2019 & 0.119 & $<\mathrm{s}$ & \\
\hline Windowing Method & 0.0099 & 0.2374 & 0.318 & $<\mathrm{s}$ & \\
\hline
\end{tabular}


The performance of the Windowing Method is very competitive concerning the average deviation (0.0099) but far poorer than $\mathrm{GA}_{1}$ concerning the Peak Error criterion ( 0.2374 for 0.0879$)$ and width of transition band (0.318 for 0.108). In contrast, the Remez (frequency transformation) approach appears to be efficient for the Peak Error (0.1026) but only moderately satisfactory for the width of transition band (0.166), and poor for the Average deviation (0.0250). It is found that the least square and Remez methods used in the transformation frequency method give results below the average. The least square method provides a slight decrease in the ripples (peak error) with respect to the Remez method but it is characterized by an increase in the width of the transition band. Considering the Peak Error, the results of $\mathrm{GA}_{2}$ and $\mathrm{GA}_{4}$ are of a poorer quality than the other genetic algorithms implemented. This indicates that the refreshing process works well only if accompanied by the selection process with diversification and adaptive mutation $\left(\mathrm{GA}_{1}\right.$ and $\left.\mathrm{GA}_{3}\right)$. The implemented mechanisms are complementary. The refreshing process is able to reseed the genetic population which leads to substantial modifications. The other mechanisms also act to increase diversification but in a moderate way that contributes to convergence.

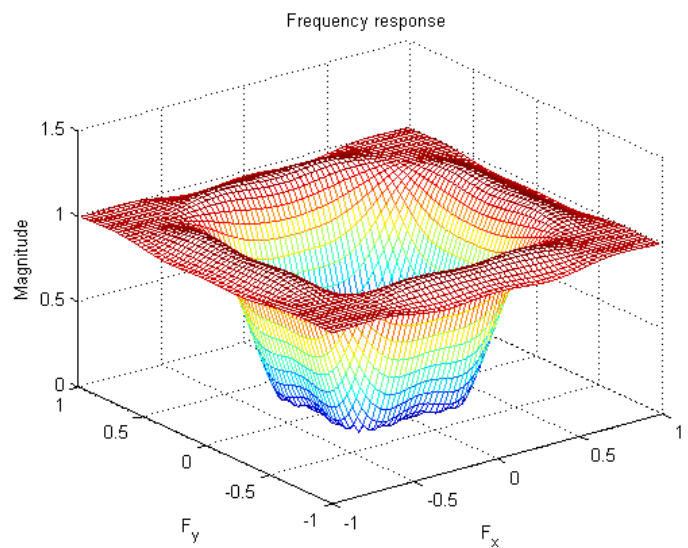

(a)

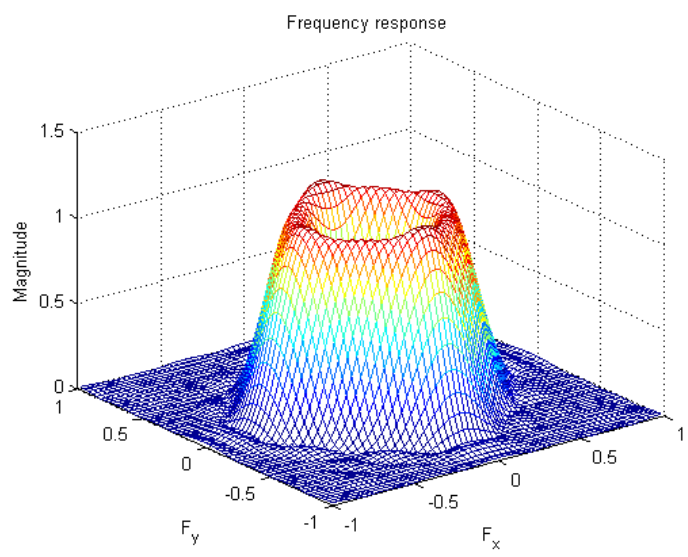

(c)

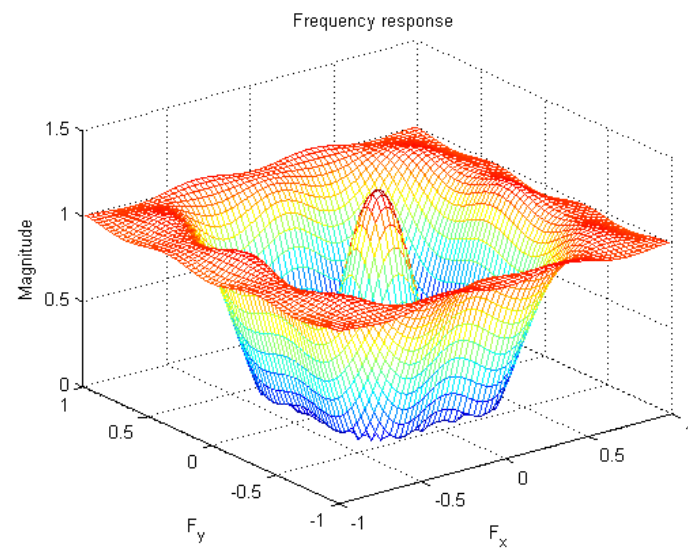

(b)

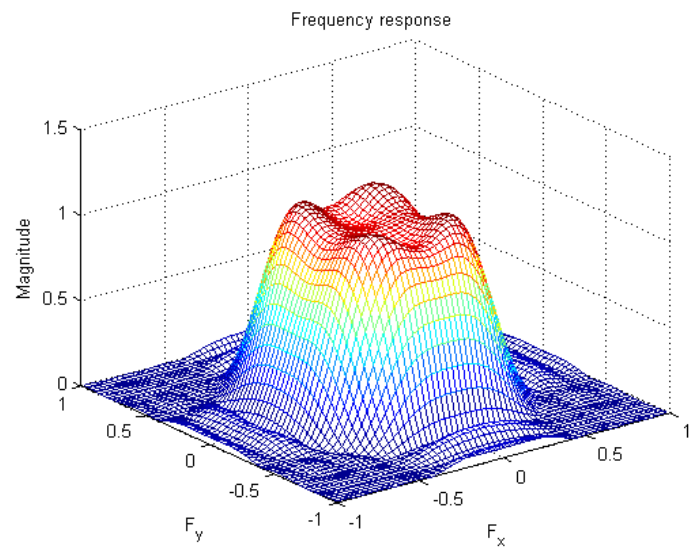

(d)

Figure 7. Frequency responses: (a) High-pass 7x7 (b) Band-stop 7x7 (c) Band-pass 9x9 (d) Low-pass $9 \times 9$.

The adaptive genetic algorithms perform better than the other techniques discussed in this paper. The Hill Climbing approach gives the worst results; this can be explained by the lack of embedded intelligence in this algorithm that runs only on the basis of a "random walk". We think that more than the approach itself, the poor results come mainly from the difficulty in tuning the input parameters. Given the lack of theoretical advances, the only way to improve the performance is to embed some intelligence via the inclusion of additional mechanisms.

Fig. 7 and 8 show the results of the application of the proposed adaptive GA $\left(\mathrm{GA}_{1}\right)$ approach to several design problems. 


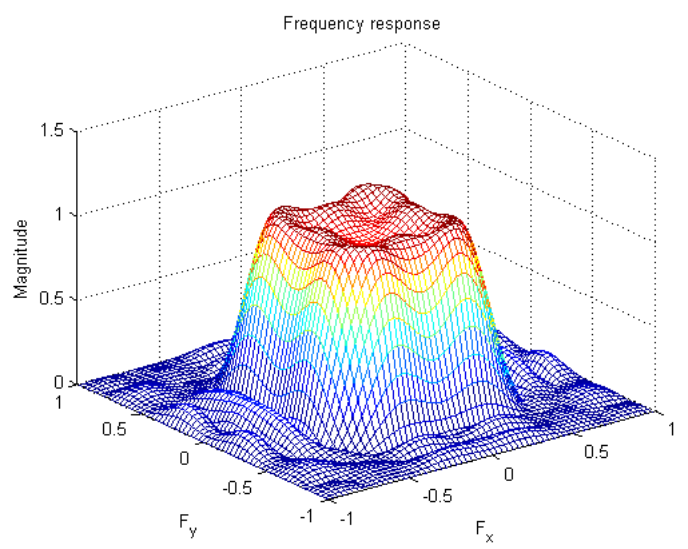

(a)

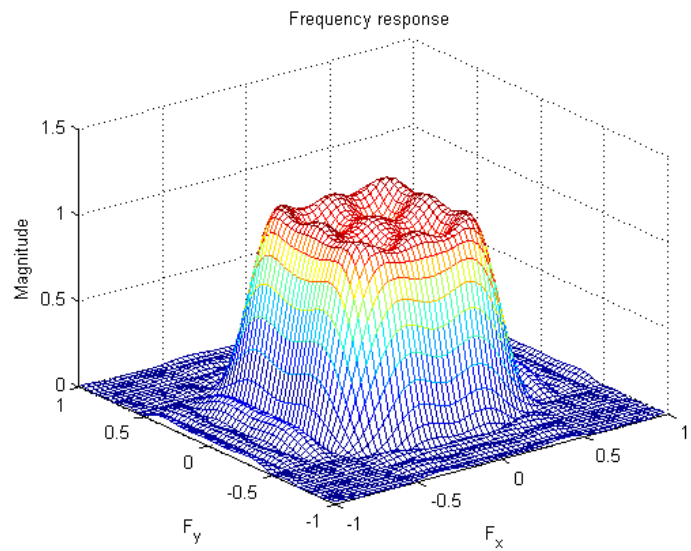

(b)

Figure 8. Frequency responses: (a) Low-pass 10x10 (b) Low-pass 11x11.

\subsection{Specific comparison with two popular genetic methods}

Among the various studies already published, those by Mastorakis et al. [31] and Tzeng [13] appear to be among the most popular and are regularly cited as references. For this reason, we propose to compare them to our approach in order to show its effectiveness. The algorithm in [13] and [31] was used to design 2-D FIR filters with two supports. The first example corresponds to the case already proposed in section 4.1. The desired magnitude response is given by Eq. 21. The second example deals with the design of a filter with the desired magnitude response (Eq. 22). We selected this case as the support is very similar to the one managed in [31].

$D 2\left(\omega_{1}, \omega_{2}\right)= \begin{cases}1 & \left|\omega_{1,2}\right| \leq 0.1 \\ 0 & \left|\omega_{1,2}\right|>0.1\end{cases}$

The algorithm of Mastorakis et al. [31] is based on a binary chromosome representation. For our trial, we developed the binary encoding version to have an exact reproduction and a real version in order to better evaluate the methodology. For the binary coded algorithm, different chromosome lengths were investigated. Each gene is coded on 10 bits, then 20 and finally 30 . Therefore, the size of chromosome depends on the size of the filter and the number of bits of genes. For the results in Tables 6 and 7, we have given the results of the genes encoding 20 bits so as not to tax the computing time. The results of encoding 20 bits and 30 bits are substantially similar, except for run time.

The results are given in Tables 6 and 7 and only $\mathrm{GA}_{1}$ performances are compared. These results show the improvement introduced by the proposed approach with respect to other successful techniques.

The results of the method used by Mastorakis et al. in [31] in the real coded version are acceptable for the example discussed in their paper. The precision of their method, however, is lower for a different filter support. The binary version appears to be ineffective for the synthesis of 2-D FIR filters, since these filters require high precision and optimization of a large number of parameters.

Table 6: Parameters of performance obtained by our proposed GA and GA approaches used in references [31] and [13] for the first example

\begin{tabular}{|c|c|c|c|c|c|}
\cline { 2 - 6 } \multicolumn{1}{c|}{} & $\begin{array}{c}\text { Error } \\
\text { (Average } \\
\text { deviation) }\end{array}$ & $\begin{array}{c}\text { Peak Error } \\
\text { (Maximum } \\
\text { deviation) }\end{array}$ & $\begin{array}{c}\text { width of } \\
\text { transition band }\end{array}$ & CPU time & Ite \\
\hline GA $_{1}$ & 0.0092 & 0.0803 & 0.113 & $49.74 \mathrm{~s}$ & 298 \\
\hline Mastorakis et al. real & 0.0289 & 0.1870 & 0.167 & $202.47 \mathrm{~s}$ & 977 \\
\hline Mastorakis et al. bin & 0.0327 & 0.2102 & 0.196 & $471.23 \mathrm{~s}$ & 1259 \\
\hline Tzeng & 0.0198 & 0.1156 & 0.156 & $140.36 \mathrm{~s}$ & 928 \\
\hline
\end{tabular}


Table 7: Parameters of performance obtained by our proposed GA and GA approaches used in references [31] and [13] for the second example

\begin{tabular}{|c|c|c|c|c|c|}
\cline { 2 - 5 } \multicolumn{1}{c|}{} & $\begin{array}{c}\text { Error } \\
\text { (Average } \\
\text { deviation) }\end{array}$ & $\begin{array}{c}\text { Peak Error } \\
\text { (Maximum } \\
\text { deviation) }\end{array}$ & $\begin{array}{c}\text { width of } \\
\text { transition band }\end{array}$ & CPU time & Ite \\
\hline $\mathrm{GA}_{1}$ & 0.0101 & 0.0924 & 0.085 & $50.31 \mathrm{~s}$ & 304 \\
\hline Mastorakis et al. real & 0.0192 & 0.1103 & 0.124 & $188.02 \mathrm{~s}$ & 930 \\
\hline Mastorakis et al. bin & 0.0292 & 0.1411 & 0.147 & $450.68 \mathrm{~s}$ & 1231 \\
\hline Tzeng & 0.0194 & 0.1116 & 0.133 & $132.25 \mathrm{~s}$ & 910 \\
\hline
\end{tabular}

The results of the method used in [13] are comparable to those obtained by the standard genetic algorithm $\left(\mathrm{GA}_{4}\right)$ mentioned in Table 4. The only difference is that several runs are required with the Tzeng algorithm to achieve the results of the standard genetic algorithm. It is not efficient with each execution of the algorithm. The results obtained are unsurprising since our mechanism has been designed in order to improve the performance of so-called standard GAs.

Concerning the Mastorakis et al. algorithm, we were initially sceptical about the binary encoding. This representation has proved to be very efficient for feature or instance selection problems for which it is completely adapted. For other cases, the question of granularity remains an issue: for a given problem, which chromosome length and input parameters should be selected for which efficiency?

In all cases, the algorithm we propose in this paper gives better results than those used in [31] and [13]. Tables 6 and 7 show that the proposed design method provides the best design results.

It is important to point out that it was difficult to find parameters leading to acceptable solutions without extensive tuning. This is a recurrent criticism leveled at evolutionary techniques.

\subsection{Additional experiments with larger filters}

The GAs have been successfully applied to various classical filters including high-pass, low-pass, band-pass and band-stop filters of different orders with the same parameter set. To illustrate the effectiveness of the proposed approach for larger filters, we consider here the design of a low pass 2-D FIR filter with dimensions $23 \times 23$, whose amplitude response is given by Eq. 22 .

The performance indices are presented in Table 8, along with those obtained with the other approaches discussed in this paper, for comparison, and indicate the better overall performance of the proposed GA approach. Again, as can be seen in Table 8, the proposed AGA $\left(\mathrm{GA}_{1}\right)$ yields better results than what has been obtained with other approaches for all the criteria.

Table 8: Parameters of performance obtained for a low-pass filter $(23 \times 23)$

\begin{tabular}{|c|c|c|c|c|c|}
\cline { 2 - 5 } \multicolumn{1}{c|}{} & $\begin{array}{c}\text { Error } \\
\text { (Average } \\
\text { deviation) }\end{array}$ & $\begin{array}{c}\text { Peak Error } \\
\text { (Maximum } \\
\text { deviation) }\end{array}$ & $\begin{array}{c}\text { width of } \\
\text { transition band }\end{array}$ & CPU time & Ite \\
\hline $\mathrm{GA}_{1}$ & 0.0087 & 0.0872 & 0.079 & $191.04 \mathrm{~s}$ & 742 \\
\hline $\mathrm{GA}_{2}$ & 0.0189 & 0.1153 & 0.126 & $235.52 \mathrm{~s}$ & 961 \\
\hline $\mathrm{GA}_{3}$ & 0.0179 & 0.1133 & 0.117 & $300.44 \mathrm{~s}$ & 1325 \\
\hline $\mathrm{GA}_{4}[18]$ & 0.0203 & 0.1185 & 0.138 & $331.18 \mathrm{~s}$ & 1608 \\
\hline Simulated Annealing [17] & 0.0216 & 0.1399 & 0.141 & $220.12 \mathrm{~s}$ & \\
\hline Tabu Search [17] & 0.0502 & 0.2208 & 0.114 & $515.86 \mathrm{~s}$ & \\
\hline Hill Climbing [17] & 0.0734 & 0.3896 & 0.108 & Random & \\
\hline $\begin{array}{c}\text { Remez (Frequency } \\
\text { Transformation) }\end{array}$ & 0.0487 & 0.1699 & 0.130 & $<\mathrm{s}$ & \\
\hline $\begin{array}{c}\text { Least Square (Frequency } \\
\text { Transformation) }\end{array}$ & 0.0396 & 0.1431 & 0.172 & $<2 \mathrm{~s}$ & \\
\hline Frequency Sampling & 0.0512 & 0.2310 & 0.105 & $<2 \mathrm{~s}$ & \\
\hline Windowing Method & 0.0371 & 0.4987 & 0.233 & $<\mathrm{s}$ & \\
\hline
\end{tabular}


Except for the convergence rate (CPU time and iteration number) which is affected by the larger dimension, the differences between the approaches concerning the other criteria are similar. For example, whereas $\mathrm{GA}_{1}$ gives an average deviation of 0.0087, the frequency sampling and windowing methods give respectively 0.0512 and 0.071. $\mathrm{GA}_{1}$ performs better at discovering relevant solutions than the other AGA versions for all criteria. This illustrates the complementary roles of the different mechanisms implemented.

\subsection{Discussion and usability studies}

This section reviews the "DNA" of our GA and demonstrates its consistency by additional experimentation.

The following set of parameters is calibrated for the class of studied filters. They are fixed for the user.

- $\quad$ Parameters related to population initialization

- Mutation parameters $k_{1}$ and $k_{2}$. They have been calibrated to be globally efficient, e.g. a good compromise between diversity and elitism.

- Crossover probability $C_{R}$

- $\quad$ Level of Mutation $P_{M}$ when a refreshing process is needed

- Parameters $\lambda_{m 1}$ and $\lambda_{m 2}$ used in the formula of mutation

- Parameters $f_{\varepsilon}, g_{\varepsilon}, f_{\text {end }}$

- $\quad$ The weight matrix $W$ to play on the ripples: the best compromise is around (5/8). It is however possible to slightly improve the performance by selecting values in the interval $(5 / 8 \pm 5 \%)$

The secondary parameters are few in number and can be considered as user parameters:

- $\Delta f_{\min }$ (equation 20)

- $\quad$ Parameters $t h_{1}, t h_{2}$ and $t h_{3}$ used to detect a local minimum (equation 17)

For these parameters, the best values are dependent on the filter size and the number of frequency points (see error calculation, equation 11). Different values were calibrated to match with different configurations (different filter size and frequency points) and they serve as references for new configurations. Their roles are perfectly understandable as they do not have any interaction with the evolutionary complexity.

In order to evaluate the usability (repeatability, performance and computational time), we generated 100 different filter types and changed the frequency cut-off ( 0.1 to 0.8$)$. For each of them, we launched 10 runs with exactly the same parameters.

The results for $\omega_{1,2}=0.2,0.3$ and 0.4 are reported in Tables 9, 10 and 11 respectively and displayed in Fig. 9.

Table 9: Parameters of performance obtained for $\omega_{1,2}=0.2$

\begin{tabular}{|c|c|c|c|c|c|c|c|c|c|c|}
\hline $\begin{array}{c}\omega_{1,2} \\
=0.2\end{array}$ & 1 & 2 & 3 & 4 & 5 & 6 & 7 & 8 & 9 & 10 \\
\hline Error & 0.0087 & 0.0087 & 0.0089 & 0.0085 & 0.0087 & 0.0090 & 0.0086 & 0.0086 & 0.0086 & 0.0087 \\
\hline Peak error & 0.0801 & 0.0784 & 0.0739 & 0.0796 & 0.0793 & 0.0828 & 0.0820 & 0.0811 & 0.0783 & 0.0801 \\
\hline CPU time & 49.87 & 46.17 & 48.79 & 51.59 & 50.20 & 50.74 & 48.03 & 49.23 & 50.02 & 50.20 \\
\hline Ite & 299 & 280 & 301 & 324 & 313 & 317 & 294 & 303 & 307 & 310 \\
\hline
\end{tabular}

Table 10: Parameters of performance obtained for $\omega_{1,2}=0.3$

\begin{tabular}{|c|c|c|c|c|c|c|c|c|c|c|}
\hline $\begin{array}{c}\omega_{1,2} \\
=0.3\end{array}$ & 1 & 2 & 3 & 4 & 5 & 6 & 7 & 8 & 9 & 10 \\
\hline Error & 0.0092 & 0.0093 & 0.0091 & 0.0094 & 0.0090 & 0.0096 & 0.0099 & 0.0093 & 0.0095 & 0.0095 \\
\hline Peak error & 0.0905 & 0.0883 & 0.0830 & 0.0847 & 0.0914 & 0.0846 & 0.0896 & 0.0845 & 0.0838 & 0.0878 \\
\hline CPU time & 50.32 & 49.93 & 48.96 & 50.73 & 51.74 & 47.30 & 52.08 & 50.29 & 49.90 & 53.19 \\
\hline Ite & 297 & 296 & 291 & 307 & 315 & 276 & 322 & 303 & 300 & 322 \\
\hline
\end{tabular}


Table 11: Parameters of performance obtained for $\omega_{1,2}=0.4$

\begin{tabular}{|c|c|c|c|c|c|c|c|c|c|c|}
\hline $\begin{array}{c}\omega_{1,2} \\
=0.4\end{array}$ & 1 & 2 & 3 & 4 & 5 & 6 & 7 & 8 & 9 & 10 \\
\hline Error & 0.0094 & 0.0096 & 0.0104 & 0.0098 & 0.0100 & 0.0093 & 0.0095 & 0.0099 & 0.0104 & 0.0099 \\
\hline Peak error & 0.0866 & 0.0826 & 0.0888 & 0.0828 & 0.0855 & 0.0832 & 0.0802 & 0.0805 & 0.0816 & 0.0899 \\
\hline CPU time & 53.69 & 49.67 & 55.51 & 51.66 & 49.72 & 49.35 & 51.86 & 52.50 & 50.04 & 51.93 \\
\hline Ite & 332 & 292 & 337 & 305 & 292 & 287 & 307 & 314 & 293 & 308 \\
\hline
\end{tabular}
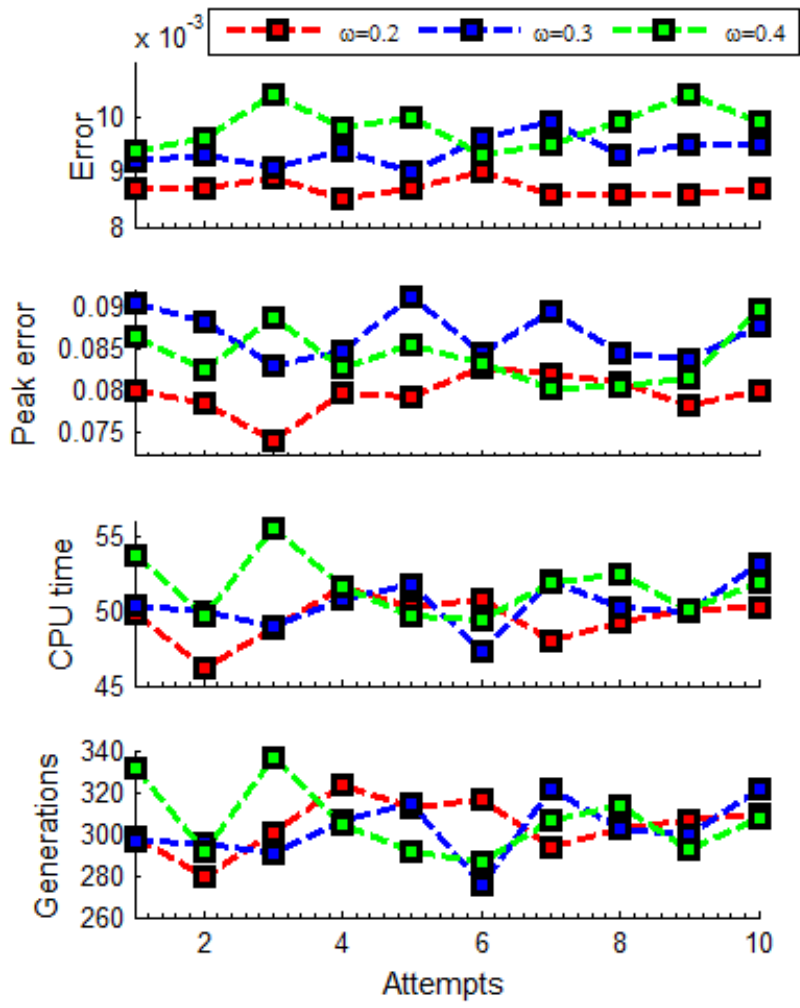

Figure 9. Results with different values of the frequency cut-off for ten runs.

To investigate further, we randomly generated 100 filters and launched the runs. Fig. 10 represents the Error and Peak Errors obtained.

The error variations (mean and peak) on large scales of tests are very small, demonstrating the consistency of our algorithm. Some cases are more distinguishable when the peak error is analyzed but the amount of variation remains very small. And for each run, the algorithm gives satisfactory results in terms of performance. 

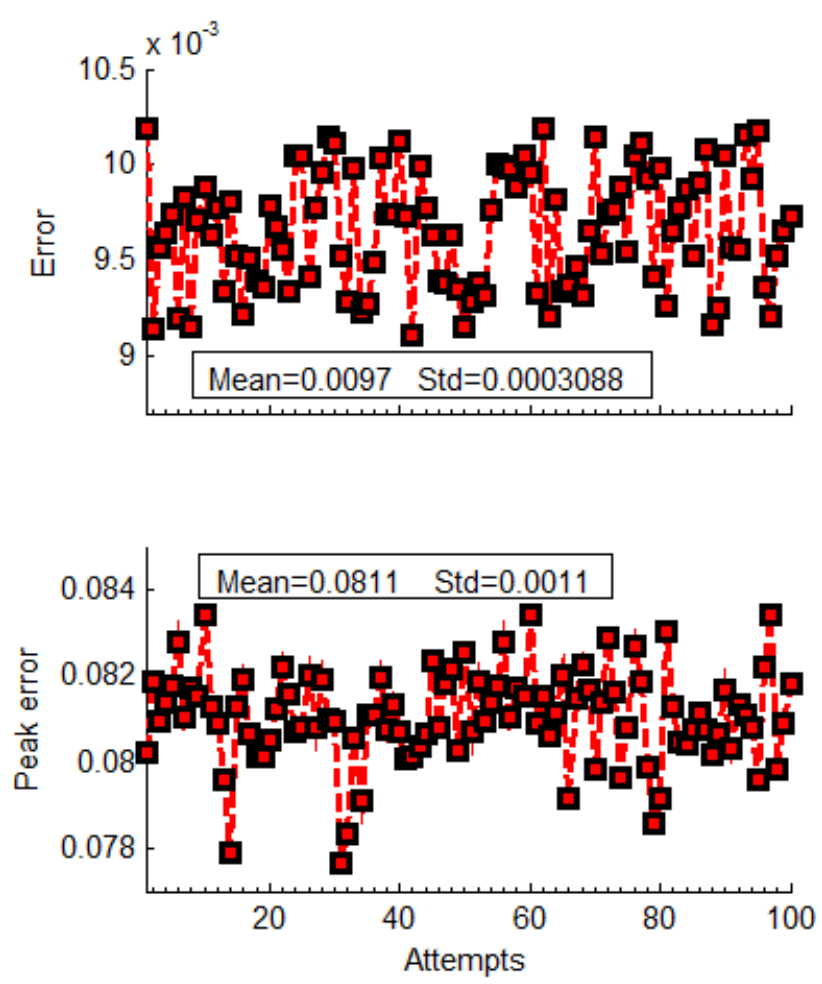

Figure 10. Errors and peak errors obtained for 100 runs.

\section{Conclusion}

This paper has presented the optimal design methods for a 2-D FIR digital filter based on the genetic algorithm. By minimizing a quadratic measure of the error in the frequency band, real-valued solutions are evolved to obtain the filter coefficients with an evolutionary algorithm. Our adaptive genetic algorithm approach dedicated for designing 2-D FIR filters can produce filters with good response characteristics while greatly reducing the error criteria and CPU time. Experiments performed with various 2-D filters have demonstrated the effectiveness and applicability of the proposed approach.

Our GA clearly outperforms classical approaches without user intervention. Fair comparison with two very popular GAs dedicated to filter design also depicts its potential. Based on two filter supports, better results were obtained with our GA with a highly reduced computational cost. More interestingly, our mechanisms afford the GA great flexibility, thus contributing to its usability which is the real challenge today. This allows a majority of parameters to be pre-calibrated, leaving only a small set user-dedicated. More than an algorithm, the "system" we suggest gives good and repeatable results, which is far from being the case with many existing solutions that are not selfadaptive enough. The user can create his own filter with confidence, as he is not faced with all the evolutionary computation complexity.

Future work will focus on the possibility of hybridizing the GA with other classic mathematical and genuinely heuristic techniques.

\section{References}

[1] X.-H. Wang, Y.-G. He, A neural network approach to FIR filter design using frequency-response masking technique, Elsevier, Signal Processing 88, 2008, pp. 2917-2926

[2] L. Cen, A hybrid genetic algorithm for the design of FIR filters with SPoT coefficients, Elsevier, Signal Processing 87, 2007, pp. 528-540

[3] J-H. Lee, S-J. Yang, D-C. Tang, Minimax design of 2-D linear-phase FIR filters with continuous and powersof-two coefficients, Elsevier, Signal Processing 80, 2000, pp. 1435-1444

[4] S-C. Pei, J-J. Shyu, Design of two-dimensional FIR digital filters by McClellan transformation and leastsquares contour mapping, Elsevier, Signal Processing 44, 1999, pp. 19-26 
[5] Y. Kamp and J. P. Thiran, Chebyshev approximation for two-dimensional nonrecursive digital filters, IEEE Trans. Circuits Syst. 22, March 1975, pp. 208-218

[6] W. S. Lu and T. Hinamoto, Optimal Design of Nonlinear-Phase FIR Filters With Prescribed Phase Error, IEEE Trans. On Sig. Processing 57( 9), 2009, pp. 3399-3410

[7] P. K. Rajan, and M. N. S. Swamy, Design of circularly symmetric two-dimensional FIR digital filters employing transformations with variable parameters, IEEE Trans. On Acoust., Speech, Signal Processing 31(3), June 1983, pp. 637-642

[8] S-C. Pei, J. J. Shyu, General form for designing two-dimensional quadrantally symmetric linear-phase FIR digital filters by analytical least-squares method , Elsevier, Signal Processing 48, 1996, pp. 165-174

[9] N. Karaboga, B. Cetinkaya, Design of digital fir filters using differential evolution algorithm, circuits systems signal processing, vol. 25, No. 5, 2006, pp. 649-660

[10] C. Charalambous, The performance of an algorithm of minimax design of two-dimensional linear phase FIR digital filters, IEEE Trans. Circuits Syst., vol. 32, Oct. 1985, pp. 1016-1028

[11] H. C. Lu and K. H. Yeh, 2-D FIR filters design using least square error with scaling-free McClellan transformation, IEEE Trans. Circuits Syst., II, vol. 47, Oct. 2000, pp. 1104-1107

[12] D. Bhattacharya, A. Antoniou, Design of 2-D FIR filters by a feedback neural network, Multidimensional Systems and Signal Processing 10, Boston, 1999, pp. 319-330

[13] S-T. Tzeng, Design of 2-D FIR digital filters with specified magnitude and group delay responses by GA approach, Signal Processing 87, 2007, pp. 2036-2044

[14] S. Sriranganathan, D.R. Bull, D.W. Redmill, Design of 2-D multiplierless FIR filters using genetic algorithms, First Int. Conf. on Genetic Algorithms in Engineering Systems: Innovations and Applications GALESIA 1995, Sheffield, UK, Sep. 1995, pp. 282-286

[15] T. Williams, M. Ahmadi, R. Hashemian, W. C. Miller, Design of High Throughput 2-D FIR Filters Using Singular Value Decomposition (SVD) and Genetic Algorithms, Proc. of the IEEE Pacific RIM Conf. on Signals, Communications and Computers, Victoria, BC, August 2001, pp. 571-574

[16] K. Boudjelaba, D. Chikouche, R.E. Bekka, Application des Algorithmes Génétiques pour la Synthèse des Filtres RIF 1-D Passe-Bas, Proceedings du 1er Congrès International sur le Génie Electrique CIGE'04, Sétif, Algeria, 10-12 Octobre 2004, pp. 36-40

[17] K. Boudjelaba, D. Chikouche, F. Ros, Evolutionary techniques for the synthesis of 2-D FIR filters, 2011 IEEE Workshop on Statistical Signal Processing (SSP'11), Nice, France, 28-30 June 2011, pp. 601-604

[18] K. Boudjelaba, F. Ros, D. Chikouche, An advanced genetic algorithm for designing 2-D FIR filters, 2011 IEEE Pacific Rim Conference on Communications, Computers and Signal Processing, Victoria, B.C., Canada, August 23-26, 2011, pp. 60-65

[19] A. Quinquis, Le Traitement du Signal sous Matlab, pratique et applications, Hermès, Paris, 2000.

[20] K-H. Yeh, H-C. Lu, Shape parameter in the two-dimensional low-pass FIR digital filters design by transformation, Elsevier, Signal Processing 53, 1996, pp. 65-74

[21] D.W. Boeringer, D.H. Werner, D.W. Machuga, A simultaneous parameter adaptation scheme for genetic algorithms with application to phased array synthesis, IEEE Transactions on Antennas and Propagation, Vol. 53, 2005, pp. 356-371

[22] F. Glover, Tabu search—part I, ORSA Journal on Computing, 1(3), 1989, pp. 190-206

[23] F. Glover, Tabu search—part II, ORSA Journal on Computing, 2(1), 1990, pp. 4-32

[24] T-H. Wu, J-Y. Yeh, C-C. Chang, A hybrid Tabu Search Algorithm to Cell Formation Problem and its Variants, World Academy of Science, Engineering and Technology 53, 2009, pp. 1090-1094

[25] S. Kirkpatrick, C.D. Gelatt, M.P. Vecchi, Optimization by Simulated Annealing, Science 220, 1983, pp. 671680

[26] I. Bohachevsky, M.E. Johnson, M.L. Stein, Generalized simulated annealing for function optimization, Technometrics 28, 1986, pp. 209-217

[27] D. Bertsimas, J. Tsitsiklis, Simulated annealing, Statistical Science, vol. 8, No. 1, 1993, pp. 10-15

[28] S.A. Dunn, The Use Of Genetic Algorithms And Stochastic Hill-Climbing In Dynamic Finite Element Model Identification, Elsevier, Computers \& Structures Vol. 66, No. 4, 1998, pp. 489-497

[29] S.A. Akramifar, G.Ghassem-Sani, Fast forward planning by guided enforced hill climbing, Elsevier, Engineering Applications of Artificial Intelligence 23, 2010, pp. 1327-1339

[30] A. W. Johnson, S. H. Jacobson, A class of convergent generalized hill climbing algorithms, Elsevier, Applied 
Mathematics and Computation 125, 2002, pp. 359-373

[31] N. E. Mastorakis, I. F. Gonos, M. N. S. Swamy, Design of Two-Dimensional Recursive Filters Using Genetic Algorithms, IEEE Transactions On Circuits And Systems I: Fundamental Theory And Applications, Vol. 50, No. 5, 2003, pp. 634-639

[32] C. R. Reeves, A Genetic Algorithm For Flowshop Sequencing, Computers and Operations Research 22 (1), 1995 , pp. 5-13

[33] J.H. Holland, Genetic algorithms and the optimal allocation of trials, SIAM Journal on Computing 2, 1973

[34] D. E. Goldberg, Genetic Algorithms in Search, Optimization, and Machine Learning, Addison-Wesley, Reading, MA, 1989

[35] F. Ros, S. Guillaume, An efficient nearest classifier, Book Chapter of Hybrid Evolutionary Systems, Studies in Computational Intelligence, Vol 75, Springer Verlag, 2007, pp. 131-147

[36] C.A. Coello, G.B. Lamont, D.A.V. Van Veldhuizen, Lamont GB, Evolutionary algorithms for solving multiobjective problems, Kluwer, New York, 2002

[37] E. Pérez, M. Posada, F. Herrera, Analysis of new niching genetic algorithms for finding multiple solutions in the job shop scheduling, J Intell Manuf 23, 2012, pp. 341-356

[38] A. Erdozain, P. M. Crespo, A new stochastic algorithm inspired on genetic algorithms to estimate signals with finite rate of innovation from noisy samples, Elsevier, Signal Processing 90, 2010, pp. 134-144

[39] J. J. Shyu, S-C. Pei, A generalized approach to the design of variable fractional-delay FIR digital filters, Elsevier, Signal Processing 88, 2008, pp. 1428-1435

[40] H-C. Lu, K-H. Yeh, Optimal design of 2-D FIR digital filters by scaling-free McClellan transformation using least-squares estimation, Elsevier, Signal Processing 58, 1997, pp. 303-308

[41] V.S. Sheeba, E. Elizabeth, Two-dimensional FIR signal adapted filter banks: Optimality and design, Elsevier, Signal Processing 87, 2007, pp. 2381-2391

[42] T. C. Speake and R. M. Mersereau, A comparison of different window formulations for twodimensional FIR filter design, IEEE Acoust., Speech, Signal Processing Conf. Rec., Apr. 1979

[43] T. C. Speake and R. M. Mersereau, A Note on the use of windows for two-dimensional FIR filter design, IEEE Trans. On Acoust., Speech, Signal Processing, vol. ASSP-29, No 1, Feb 1981, pp. 125-127

[44] D. T. Nguyen and M. N. S. Swamy, Approximation design of 2-D digital filters with elliptical magnitude response of arbitrary orientation, IEEE Trans. Circuits Syst., vol. 33, June 1986, pp. 597-603

[45] W.-P. Zhu, M. 0. Ahmad, and M. N. S. Swamy, A closed-form solution to the least-square design problem of 2-D linear-phase FIR filters, IEEE Trans. Circuits Syst., II, vol. 44, Dec 1997, pp. 1032-1039

[46] S. Nordebo and I. Claesson, Minimum Norm Design of Two-Dimensional Weighted Chebyshev FIR Filters, IEEE Trans. Circuits Syst.-II, vol. 44, No. 3, Mar. 1997, pp. 251-253

[47] W.-P. Zhu, M. 0. Ahmad, and M. N. S. Swamy, Realization of 2-D Linear-Phase FIR Filters by Using the Singular-Value Decomposition, IEEE Trans. On Sig. Processing, vol. 47, No 5, May 1999, pp. 1349-1358

[48] S. C. Pei and P. H. Wang, Design of Arbitrary Cutoff 2-D Diamond-Shaped FIR Filters Using the Bernstein Polynomial, IEEE Sig. Processing Letters, vol. 7, No 11, Nov 2000, pp. 310-313

[49] W.-S. Lu, A unified approach for the design of 2-D digital filters via semidefinite programming, IEEE Trans. Circuits Syst., vol. 49, June 2002, pp. 814-826

[50] W.-S. Lu and T. Hinamoto, A new minimax design for 2-D FIR filters with low group delay, ISCAS'2005, Kobe, Japan, May 2005, pp. 2028-2031

[51] W.-S. Lu and T. Hinamoto, A Second-Order Cone Programming Approach for Minimax Design of 2-D FIR Filters with Low Group Delay, ISCAS 2006, Island of Kos, Greece, May 2006, pp. 2521-2524

[52] W.-S. Lu and T. Hinamoto, Design of FIR filters with discrete coefficients via polynomial programming: Towards the global solution, ISCAS 2007, New Orleans, May 2007, pp. 2048-2051 